\title{
An Amygdalo-Parabrachial Pathway Regulates Pain Perception and Chronic Pain
}

\author{
${ }^{\circledR}$ Charles Raver, ${ }^{1}$ Olivia Uddin, ${ }^{1,3}$ Yadong Ji, ${ }^{2}$ Ying Li, ${ }^{1}$ Nathan Cramer, ${ }^{1}$ Carleigh Jenne, ${ }^{1}$ Marisela Morales, ${ }^{4}$ \\ Radi Masri, ${ }^{1,2}$ and ${ }^{\circledR}$ Asaf Keller ${ }^{1,3}$ \\ ${ }^{1}$ Department of Anatomy \& Neurobiology, University of Maryland School of Medicine, Baltimore, Maryland 21201, ${ }^{2}$ Department of Advanced Oral \\ Sciences and Therapeutics, University of Maryland School of Dentistry, Baltimore, Maryland 21201, ${ }^{3}$ Program in Neuroscience, University of \\ Maryland School of Medicine, Baltimore, Maryland 21201, and ${ }^{4}$ Neuronal Networks Section, Intramural Research Program, National Institute on \\ Drug Abuse, Baltimore, Maryland 21224
}

The parabrachial $(\mathrm{PB})$ complex mediates both ascending nociceptive signaling and descending pain modulatory information in the affective/emotional pain pathway. We have recently reported that chronic pain is associated with amplified activity of PB neurons in a rat model of neuropathic pain. Here we demonstrate that similar activity amplification occurs in mice, and that this is related to suppressed inhibition to lateral parabrachial (LPB) neurons from the CeA in animals of either sex. Animals with pain after chronic constriction injury of the infraorbital nerve (CCI-Pain) displayed higher spontaneous and evoked activity in PB neurons, and a dramatic increase in after-discharges, responses that far outlast the stimulus, compared with controls. LPB neurons in CCI-Pain animals showed a reduction in inhibitory, GABAergic inputs. We show that, in both rats and mice, LPB contains few GABAergic neurons, and that most of its GABAergic inputs arise from CeA. These CeA GABA neurons express dynorphin, somatostatin, and/or corticotropin releasing hormone. We find that the efficacy of this CeA-LPB pathway is suppressed in chronic pain. Further, optogenetically stimulating this pathway suppresses acute pain, and inhibiting it, in naive animals, evokes pain behaviors. These findings demonstrate that the CeA-LPB pathway is critically involved in pain regulation, and in the pathogenesis of chronic pain.

Key words: dynorphin; CRH; GABA; parabrachial; central amygdala

Significance Statement

We describe a novel pathway, consisting of inhibition by dynorphin, somatostatin, and corticotropin-releasing hormoneexpressing neurons in the CeA that project to the parabrachial nucleus. We show that this pathway regulates the activity of pain-related neurons in parabrachial nucleus, and that, in chronic pain, this inhibitory pathway is suppressed, and that this suppression is causally related to pain perception. We propose that this amygdalo-parabrachial pathway is a key regulator of both chronic and acute pain, and a novel target for pain relief.

\section{Introduction}

Chronic pain profoundly affects quality of life (Dworkin et al., 2007; O'Connor and Dworkin, 2009; van Hecke et al., 2014) and

Received Jan. 10, 2020; revised Mar. 9, 2020; accepted Mar. 11, 2020.

Author contributions: C.R., M.M., R.M., and A.K. designed research; C.R., O.U., Y.J., Y.L., N.C., C.J., M.M., R.M., and A.K. performed research; C.R., O.U., Y.J., Y.L., N.C., C.J., M.M., and A.K. analyzed data; C.R., O.U., N. C., M.M., R.M., and A.K. edited the paper; C.R. and A.K. wrote the paper.

This work was supported by National Institutes of Health National Institute of Neurological Disorders and Stroke Grants R01NS099245 and R01NS069568. The content is solely the responsibility of the authors and does not necessarily represent the official views of the National Institutes of Health. The funding sources had no role in study design; the collection, analysis and interpretation of data; the writing of the report; or in the decision to submit the article for publication. We thank Dr. Adam Puche for sharing histological expertise; and Andrew Furman for sharing MATLAB routines.

The authors declare no competing financial interests.

Correspondence should be addressed to Asaf Keller at akeller@som.umaryland.edu.

https://doi.org/10.1523/JNEUROSCI.0075-20.2020

Copyright $\odot 2020$ the authors afflicts over 100 million people, costing up to $\$ 650$ billion a year in medical treatment and lost productivity in the United States alone (Institute of Medicine Committee on Advancing Pain Research, Care, and Education, 2011). Chronic pain is the most common complaint of patients in outpatient clinics (Upshur et al., 2006), and effective therapies remain limited (Meyer-Rosberg et al., 2001; Dworkin et al., 2013; Finnerup et al., 2015). More importantly, these therapies lack specificity to the underlying pathophysiology and carry a high burden of side effects for pain patients (Manion et al., 2019).

Attempts to treat pain have often ignored the role of cognitive, attentional, and emotional aspects of pain perception in favor of traditional pharmaceutical approaches (Bushnell et al., 2013). While animal models have provided indirect evidence, studies in human participants directly demonstrate the ability of emotional and attentional factors to modify affective dimensions of pain (Loggia et al., 2008; Roy et al., 2008) and potentially the 
sensory-discriminatory aspects of pain (deCharms et al., 2005; Roy et al., 2011). Moreover, there is growing evidence that therapies targeting the motivational-cognitive dimensions of pain may be more promising (Auvray et al., 2010). Indeed, there is increasing evidence that the negative affective, cognitive, and psychosocial state of chronic pain is universal in different chronic pain states (Gustin et al., 2011). Therefore, understanding the role of the affective-motivational pathways in chronic pain may lead to innovative therapies to treat these widespread conditions.

A key structure for encoding the affective component of pain is the parabrachial complex (PB). PB comprises the KöllikerFuse nucleus and the lateral and medial PB nuclei. It is located at the midbrain-pons junction and derives its name from its proximity to the superior cerebellar peduncle. PB has extensive, often reciprocal, connections with brainstem and forebrain structures, and it plays key roles in functions, such as satiety and appetite, sleep and arousal, cardiovascular function, and fluid homeostasis (Hajnal et al., 2009; Martelli et al., 2013; Davern, 2014). Importantly, $\mathrm{PB}$ plays a prominent role in pain processing (Gauriau and Bernard, 2002; Roeder et al., 2016; Uddin et al., 2018; Chiang et al., 2019).

PB receives dense inputs from lamina I nociceptive spinal neurons, a projection far denser than the spinothalamic pathway (Spike et al., 2003; Polgár et al., 2010). As a result, PB neurons can respond robustly, and preferentially, to noxious stimuli (Gauriau and Bernard, 2002; Uddin et al., 2018). PB projects, often reciprocally, to several regions linked to pain and affect, including the periaqueductal gray, rostroventral medulla, thalamus, amygdala, and zona incerta (Bianchi et al., 1998; Roeder et al., 2016). Thus, $\mathrm{PB}$ appears to serve as a key nexus for pain and its affective perception.

PB is involved also in chronic pain. Matsumoto et al. (1996) reported that $\mathrm{PB}$ neuronal activity is increased in a rat model of arthritic pain. Expression of immediate early genes in $\mathrm{PB}$ increases after chronic constriction injury (CCI) of the sciatic nerve in rats (Jergova et al., 2008). We demonstrated that chronic pain after CCI of the infraorbital nerve (CCI-ION) is associated with amplified activity of PB neurons (Uddin et al., 2018). This amplified activity was expressed as a dramatic increase in afterdischarges (ADs), the excessively prolonged neuronal responses that far outlast a sensory stimulus. Because these ADs are likely causally related to chronic pain (Laird and Bennett, 1993; Asada et al., 1996; Okubo et al., 2013), it is important to understand the mechanisms leading to their generation, and the circuitry involved in their pathophysiology.

Here, we describe a novel pathway, the CeA-LPB pathway, which potently inhibits $\mathrm{PB}$ neurons. This inhibition regulates $\mathrm{PB}$ responses to nociceptive inputs, and dysregulation of this inhibition leads to amplified activity of $\mathrm{PB}$ neurons, and to chronic pain.

\section{Materials and Methods}

We adhered to accepted standards for rigorous study design and reporting to maximize the reproducibility and translational potential of our findings as described by Landis et al. (2012) and in ARRIVE (Animal Research: Reporting In Vivo Experiments). In line with National Institutes of Health recommendations for scientific rigor, we performed an a priori power analysis to estimate required sample sizes (Landis et al., 2012).

\section{Subjects}

All procedures adhered to Animal Welfare Act regulations, Public Health Service guidelines, and approved by the University of Maryland
School of Medicine Animal Care and Use Committee. We studied male and female adult rats and mice, unless otherwise noted. We studied 14 Sprague Dawley rats (acquired from Envigo), 11 corticotropin releasing hormone-Cre (CRH-Cre) rats (generously provided by R. Messing, University of Texas, Austin) bred on a Wistar background, 43 WT Wistar rats (from our breeding colony, founding breeders acquired from Envigo), 3 GAD2-GFP mice (generously provided by A. Puche, University of Maryland, Baltimore) bred on a B6CBAF1/J (The Jackson Laboratory) background, 21 GAD2-IRES-Cre mice (The Jackson Laboratory), and 15 C57Bl6/J background mice (from our breeding colony, founding breeders from The Jackson Laboratory).

\section{Recovery surgical procedures}

Induction of chronic orofacial pain

We used a rodent model of neuropathic pain, evoked by CCI-ION (Vos et al., 1994; Benoist et al., 1999; Okubo et al., 2013; Akintola et al., 2017; Castro et al., 2017a). We first anesthetized animals with isoflurane, followed by ketamine/xylazine (i.p.). We made intraoral incisions along the roof of the mouth next to left cheek, beginning distal to the first molar. We freed the infraorbital nerve from surrounding connective tissue before loosely tying it with silk thread (4-0), 1-2 mm from its emergence from the infraorbital foramen. After the surgery, we monitored the animals daily as they recovered for 5-7 d in their home cage.

Viral construct and anatomic tracer injections, iontophoresis, and cannula implants

We placed animals, deeply anesthetized with isoflurane, in a stereotaxic frame and created a small craniotomy over the ROI, targeting those regions under stereotaxic guidance. For delivery of viral constructs to the CeA, we injected $0.8-1 \mu \mathrm{l}$ of the viral construct at a rate of $50 \mathrm{nl} / \mathrm{min}$, using glass pipettes (40-60 $\mu \mathrm{m}$ tip diameter), coupled to a Hamilton syringe controlled by a motorized pump. The pipette was left in place for $10 \mathrm{~min}$ before being slowly retracted over 5-10 min. We targeted the CeA using the following coordinates: in rats (AP $-2.2 \mathrm{~mm}$ and $\mathrm{ML} 4.3$ $\mathrm{mm}$, relative to bregma, and DV $-6.7 \mathrm{~mm}$ relative to dural surface) and in mice (AP $-1.1 \mathrm{~mm}$ and $\mathrm{ML} 2.6 \mathrm{~mm}$, relative to bregma, and DV $-4.0 \mathrm{~mm}$ relative to dural surface).

For delivery of retrograde anatomic tracers to the $\mathrm{PB}$ of rats and mice, we used either cholera toxin subunit B (CTB, List Biological Labs) or Fluoro-Gold (FG; Fluorochrome), as noted in Results. We injected $1 \mu \mathrm{l}$ of $0.5 \%$ CTB (List Biological Labs) in saline through glass pipettes (tip diameter $40 \mu \mathrm{m}$ ) at a rate of $50 \mathrm{nl} / \mathrm{min}$. For the delivery of FG, we iontophoretically injected $3 \%$ tracer dissolved in $0.1 \mathrm{M}$ cacodylate buffer using glass pipettes (10-15 $\mu \mathrm{m}$ tips). We delivered pulses of $5 \mu \mathrm{A}$ (positive polarity, 5-7 s duty cycle, $30 \mathrm{~min}$ ). We targeted $\mathrm{PB}$ using the transverse sinus and the following coordinates: in rats (AP -8.6 to $-9.0 \mathrm{~mm}$ and ML $2.0 \mathrm{~mm}$, relative to bregma, and DV $-5.3 \mathrm{~mm}$ relative to dural surface) and in mice (AP $-4.6 \mathrm{~mm}$ and ML $1.2 \mathrm{~mm}$, relative to bregma, and DV $-3.2 \mathrm{~mm}$ relative to dural surface).

To pharmacologically or optogenetically manipulate $\mathrm{PB}$, we implanted either stainless-steel guide cannulas (26G, Plastics One) or fiberoptic cannulas $(200 \mu \mathrm{m}$ core, 0.37NA, RWD Life Science) using coordinates listed above for rats. Throughout the postimplantation recovery period, we handled animals regularly to acclimate them to removal of the cannula's protective cap and connection to the internal cannula or fiberoptic patch cable.

\section{Behavioral assessment of pain and hyperalgesia}

Mechanical sensitivity

To assess tactile sensitivity, we held animals loosely without restraint on the experimenter's arm (rats) or hand (mice), while von Frey filaments (North Coast Medical) of varying forces were applied to the buccal region. We tested each animal bilaterally, and a response was defined as an active withdrawal of the head from the probing filament. We used the up-down method to determine withdrawal thresholds, as described previously (Dixon, 1965; Chaplan et al., 1994; Akintola et al., 2017). We used the same approach to assess tactile responses on the plantar surface of the hindpaws, in animals standing on a metal grid. We compared 
grouped data with Mann-Whitney $U$ ranked-sum tests or Friedman's nonparametric repeated-measures tests as noted (see Results).

\section{Dynamic allodynia}

To assess dynamic mechanical sensitivity, we performed a modified brush test (adapted from Cheng et al., 2017). Briefly, we placed animals in a clear Plexiglas chamber on a raised wire platform to provide unrestricted access to the hindpaw. One round consisted of three brush stimuli applied to the lateral plantar surface of the hindpaw at intervals of 10 s. We performed three rounds, alternating left and right hindpaws with 3 min between rounds. The stimuli consisted of a light stroke from proximal to distal end of the plantar surface, using a clean \#4 paintbrush trimmed flat. We counted the number of responses of 9 total stimulus applications for each hindpaw. A response consisted of flicking, licking, or a complete lifting of the hindpaw from the floor, whereas we did not count only partial movement of the paw or limb that did not result in the paw lifting from the platform.

\section{Grimace scale}

To assess ongoing pain, we analyzed facial grimace behavior (Langford et al., 2010; Sotocinal et al., 2011; Akintola et al., 2017, 2019). We placed animals in a square Plexiglas chamber $(6 \times 8$ inches for rats, $3 \times 5$ inches for mice) with two opaque sides and two transparent sides on a raised wire platform. For all experiments, except optogenetic stimulation, we recorded each animal for $30 \mathrm{~min}$, with cameras facing each of the transparent sides of the chamber. For the optogenetics experiments, we placed animals in a modified Plexiglas chamber with three transparent sides, and recorded throughout the mechanical sensitivity tests.

We scored facial expressions via a semiautomated procedure using the "Face Finder" application (Sotocinal et al., 2011) generously gifted by J. Mogil. We screened, labeled, scrambled, and scored face images with the experimenter blinded to the treatment group and identity of each image using a custom MATLAB (The MathWorks) script. The grimace scale quantifies changes in four action units (AUs): orbital tightening, nose-cheek bulge, whisker tightening, and ear position. We selected 10 screenshots for each animal or each time point (during within-subject and repeated-measures tests); and on each image, each AU was given a score of 0 , 1, or 2, as previously described (Langford et al., 2010; Sotocinal et al., 2011; Akintola et al., 2017, 2019). We calculated mean grimace scale scores as the average score across all the AUs.

\section{In vivo electrophysiology}

Surgical preparation

We lightly anesthetized (Level III-2, as defined by Friedberg et al., 1999) C57Bl6/J mice with $20 \%$ urethane. We placed mice in a stereotaxic frame, with body heat maintenance, and made a small craniotomy over the recording site to target $\mathrm{PB}$ ( $\mathrm{AP}-4.0$ to $-4.6 \mathrm{~mm}$ and $\mathrm{ML}$ 1.2-1.5 $\mathrm{mm}$, relative to bregma, and DV -3.0 to $-3.5 \mathrm{~mm}$, relative to dural surface). Post hoc histologic analysis and small electrolytic lesions confirmed all recording sites; cells falling outside of $\mathrm{PB}$ were excluded. To identify recording sites, electrolytic lesions were made at the end of a recording session. We sectioned the fixed brain tissue into $80-\mu \mathrm{m}$-thick coronal sections that were stained with cresyl violet.

\section{Electrophysiological recording}

Using platinum-iridium recording electrodes (2-4 M $\Omega$ ) produced in our laboratory, we recorded from PB ipsilateral to CCI-ION. We isolated units responsive to noxious cutaneous stimuli, to dermatomes in both the head and body, and digitized the waveforms using a Plexon system (Plexon). Upon encountering a neuron responsive to noxious cutaneous stimulation, we allowed the neuron to resume baseline firing rate before recording spontaneous activity for $3 \mathrm{~min}$, after which we recorded neuronal responses to noxious stimuli. We applied mechanical stimuli within the V2 dermatome, or to the plantar surface of the hindpaw. Mechanical stimulation was produced with calibrated electronic forceps. We applied five repetitions each of mechanical stimulus, alternating between ipsilateral and contralateral receptive fields. When the neuron recorded resumed firing at its baseline rate, we applied stimuli with at least $8 \mathrm{~s}$ between each application. If neurons exhibited ADs, we extended the interstimulus interval to capture the entire AD duration.

\section{Electrophysiology data analysis}

We sorted neurons using Offline Sorter (Plexon) using dual thresholds and principal component analysis. We subsequently generated autocorrelograms in NeuroExplorer (Plexon) to confirm that each recording was of a single unit. We analyzed responses to tactile stimuli using custom MATLAB routines, written to calculate the integral of force applied by the forceps, the firing rate during stimulus application, and the spontaneous firing rate. Evoked responses were computed and expressed as evoked firing rate normalized to spontaneous firing rate, divided by the stimulus force integral (Castro et al., 2017b).

We defined ADs, periods of sustained activity that outlast a stimulus presentation (Okubo et al., 2013; Uddin et al., 2018), as peristimulus time histogram bins in which activity exceeded the $99 \%$ CI for a period lasting at least $500 \mathrm{~ms}$ after stimulus offset.

\section{In vitro electrophysiology}

Surgical preparation of animals

At least 3 weeks before in vitro electrophysiological recordings, we injected CeA of GAD-Cre mice (21-30 d old) with channelrhodopsin viral constructs, as described above, AAV-EF1a-DIO-hChR2(H134R)EYFP (UNC Vector Core, Chapel Hill, NC). Animals recovered for 5-7 d from injection of the viral construct before being assessed for baseline mechanical sensitivity. Animals underwent CCI-ION surgery (as described above) or sham surgery, and were allowed to recover for an additional 2 weeks. Just before electrophysiological recordings, we measured mechanical sensitivity thresholds to confirm the presence or absence of CCI-ION pain.

\section{In vitro recordings}

We anesthetized animals with ketamine/xylazine, removed their brains, and prepared horizontal slices $(300-\mu \mathrm{m}$-thick) containing PB, following the method described by Ting et al. (2014). For recordings, we placed slices in a submersion chamber and continually perfused $(2 \mathrm{ml} / \mathrm{min})$ with ACSF containing the following (in $\mathrm{mM}$ ): $119 \mathrm{NaCl}, 2.5 \mathrm{KCl}, 1.2$ $\mathrm{NaH}_{2} \mathrm{PO}_{4}, 2.4 \mathrm{NaHCO}_{3}, 12.5$ glucose, $2 \mathrm{MgSO}_{4} \cdot 7 \mathrm{H}_{2} \mathrm{O}$, and 2 $\mathrm{CaCl}_{2} \cdot 2 \mathrm{H}_{2} \mathrm{O}$.

We obtained whole-cell patch-clamp recordings, in voltage-clamp mode, through pipettes containing the following (in $\mathrm{mM}$ ): 130 cesium methanesulfonate, 10 HEPES, 1 magnesium chloride, 2.5 ATP-Mg, 0.5 EGTA, and 0.2 GTP-Tris. For recordings in bridge mode, we replaced cesium methanesulfonate with potassium gluconate $(120 \mathrm{~mm})$ and potassium chloride $(10 \mathrm{~mm})$. Impedance of patch electrodes was 4-6 $\mathrm{M} \Omega$. Series resistance $<40 \mathrm{M} \Omega$ was monitored throughout the recording, and recordings were discarded if series resistance changed by $>20 \%$. All recordings were obtained at room temperature.

To optically activate ChR2, we collimated blue light through a waterimmersion $40 \times$ microscope objective to achieve whole-field illumination. Light source was a single wavelength $(470 \mathrm{~nm})$ LED system (CoolLED pE-100, Scientifica), controlled through a TTL signal.

\section{Pharmacological and optogenetic manipulation of $\mathrm{PB}$}

Inhibition of $P B$

To assess whether inhibiting PB can alleviate CCI-ION pain, we first implanted WT Wistar rats with a single guide cannula above the right $\mathrm{PB}$, as described above. Two weeks later, we recorded baseline facial and hindpaw mechanical withdrawal thresholds and rat grimace scale (RGS) scores. Animals then underwent CCI-ION surgery; and, 2 months later, we obtained mechanical and spontaneous ongoing pain scores. We randomly assigned animals to a saline or muscimol group, and infused $50 \mu \mathrm{L}$ of $50 \mu \mathrm{m}$ muscimol, or saline, at $2.5 \mu \mathrm{L} / \mathrm{min}$, into $\mathrm{PB}$ via internal cannula temporarily inserted into the chronic guide cannula. We immediately tested mechanical withdrawal thresholds and RGS scores following drug infusion. After 1 week to allow for drug washout, we switched the treatment groups (drug/saline) and animals were retested. We confirmed cannula implantation locations by post hoc histologic analysis. 


\section{Disinhibition of the $P B$}

To assess whether disinhibiting PB can evoke behavioral metrics of pain, we implanted WT Wistar rats with a single cannula in the right $\mathrm{PB}$, similar to the muscimol experiments above. Animals recovered for 2 weeks after implantation before testing baseline hindpaw mechanical withdrawal thresholds. We randomly assigned animals to saline or gabazine groups, and infused $50 \mu \mathrm{l}$ of $200 \mathrm{~nm}$ gabazine, or saline, at $2.5 \mu \mathrm{l} / \mathrm{min}$ into PB. We immediately tested hindpaw mechanical withdrawal thresholds. We normalized each animal's postdrug behavioral score to its baseline score.

\section{Optogenetic activation of the CeA-PB terminals}

To determine whether selectively activating the CeA-PB pathway can alter pain sensation, we injected WT Wistar or CRH-Cre rats with channelrhodopsin viral constructs, and implanted fiberoptic cannulas, as described above. We injected AAV-hSyn-hChR2(H134R)-mCherry (or AAV-hSyn-hChR2(H134R)-EYFP) and AAV-EF1a-DIO-hChR2 (H134R)-EYFP into the right CeA of WT Wistar and CRH-Cre rats, respectively. In all animals, we implanted a single fiberoptic cannula over the right $\mathrm{PB}$. Animals recovered for 9 weeks before behavioral testing. Although fluorescent reporter protein expression can be observed in $\mathrm{PB}$ starting at $\sim 6$ weeks after injection, expression appears to peak and stabilize at $\sim 8-9$ weeks.

We tested all fiberoptic cannulas before implantation and calibrated the laser light source at the beginning of each testing session to deliver 4 $\mathrm{mW}$ of $470 \mathrm{~nm}$ light at the fiberoptic terminus. We stimulated CeA-PB terminals with short trains of 5 stimuli $(4 \mathrm{~ms}$ duration/stimulus, $10 \mathrm{~Hz}$ ) from a $470 \mathrm{~nm}$ laser controlled by TTL pulse from Master- 8 pulse generator (A.M.P.I.). The experimenter triggered each train of light manually, with a $5 \mathrm{~s}$ delay, to ensure application of mechanical and dynamic hindpaw stimuli could be time locked with light delivery. During all testing sessions, we connected animals to the fiberoptic patch cable and held constant other behavioral cues with the light stimulation portion of the testing session.

After the 9 week recovery, we collected baseline mechanical sensitivity, dynamic mechanical sensitivity, and RGS scores. In naive animals, we performed dynamic mechanical test with animals connected to the fiberoptic patch cable without light delivery, waited for a $5 \mathrm{~min}$ recovery period, and repeated the test with delivery of light time-locked to each hindpaw stimulus. We then returned animals to their home cage and allowed a 90-120 min rest period. We then performed the mechanical withdrawal test in the same manner: hindpaw stimuli applied but no light delivery, $5 \mathrm{~min}$ rest period, hindpaw stimuli applied with concurrent light delivery. For the acute formalin pain test, we repeated this same testing paradigm after injecting $50 \mu \mathrm{l}$ of $5 \%$ formalin (Sigma Millipore), injected subcutaneously into the dorsal surface of the left hindpaw (contralateral to injection and cannula implantation). We waited $\sim 90 \mathrm{~min}$ to allow the initial pain response, lifting, licking, and guarding of the hindpaw, to subside before starting the mechanical sensitivity tests. We videotaped every session to gather RGS scores with and without optogenetic activation of the CeA-PB. We collected RGS scores in this manner to avoid prolonged activation of this pathway in the absence of a pain stimulus, as well as the confounding effects of prolonged blue light exposure (Tyssowski and Gray, 2019).

\section{Anatomical, immunohistochemical, and neurochemical techniques Tissue preparation}

After a postinjection recovery period, specific to the tracer and species (see below), we deeply anesthetized animals with ketamine/xylazine, or urethane, and perfused them transcardially with PBS, followed by $4 \%$ PFA (Sigma Millipore). We removed brains and immersed them in fixative overnight at $4^{\circ} \mathrm{C}$. Using a vibrating microtome $(50 \mu \mathrm{m}$ sections) or a cryostat (14-16 $\mu \mathrm{m}$ sections), we collected coronal sections through CeA and $\mathrm{PB}$.

\section{Anterograde viral tracing}

We injected CeA of GAD-Cre mice and CRH-Cre rats with AAV-EF1aDIO-hChR2-EYFP viral constructs (see above). After at least 3 weeks for mice and 9 weeks for rats, we perfused, collected, and sectioned tissue.
We rinsed free-floating sections $(50 \mu \mathrm{m})$ of the injection site $(\mathrm{CeA})$ and projection site $(\mathrm{PB})$ several times in PBS, and counterstained with DAPI $(100 \mathrm{ng} / \mathrm{ml}$ for $5 \mathrm{~min})$. We then mounted and coverslipped tissue with a nonfluorescing hydrophilic mounting media.

\section{Identification of inhibitory neurons in $P B$}

We collected brains from formalin-perfused Wistar rats, sectioned (14 $\mu \mathrm{m}$ thick) and mounted frozen sections directly onto Superfrost Plus slides (Thermo Fisher Scientific). We processed sections for mRNA transcripts encoding the vesicular GABA transporter (VGAT) using RNAscope (Advanced Cell Diagnostics). According to the manufacturer's directions, we treated sections with heat, protease digestion, and hybridization of the target probes with Opal 520 fluorophore (PerkinElmer). Following hybridization of the fluorophore via RNAscope, we treated all sections for NeuN immunoreactivity using rabbit anti-NeuN antibody (Abcam; ab128886; 1:500). We blocked sections for $30 \mathrm{~min}$ at room temperature in $2 \%$ normal donkey serum with $0.2 \%$ Triton X-100, followed by incubation in rabbit anti-NeuN for $24 \mathrm{~h}$ at $4^{\circ} \mathrm{C}$. We washed sections twice in PBS, followed by incubation with Cy3-conjugated donkey anti-rabbit (Jackson ImmunoResearch Laboratories; \#711-165-152; 1:300) for $1 \mathrm{~h}$ at room temperature. We washed sections twice in PBS, counterstained with DAPI, and coverslipped with ProLong Gold mounting medium (Invitrogen). Each batch included sections processed with negative and positive control probes to ensure specificity of the target probes.

Following RNAscope and NeuN immunolabeling, we quantified VGAT-expressing LPB neurons from images photographed with a Leica Microsystems TCS SP8 confocal microscope. We held excitation and detection parameters constant across all sections. We imaged full sections using a $5 \times$ objective, and LPB and surrounding structures using $40 \times$ oil immersion objective. We reconstructed sections using Leica LAS $\mathrm{X}$ Navigator tiling software. Finally, we analyzed sections using StereoInvestigator (MBF Bioscience) after drawing boundaries for LPB (Paxinos and Watson, 2013). In each section, we identified and quantified VGAT and NeuN-positive cells by dividing LPB in to $100 \mu \mathrm{m} \times$ $100 \mu \mathrm{m}$ grids and sampling $25 \%$ of each grid. We analyzed the left and right LPB of four sections spaced $280 \mu \mathrm{m}$ apart from each animal.

\section{Retrograde immunohistochemical identification of CeA-PB neurons}

We injected PB of GAD-GFP mice and Sprague Dawley rats with CTB (see above). Between 7 and $14 \mathrm{~d}$ after injection, we perfused, collected, and sectioned tissue. We processed free-floating sections $(50 \mu \mathrm{m})$ for double-label immunohistochemistry with antibodies against CTB (goat anti-CTB; List Biological Labs; product \#703; 1:20,000) and against calbindin (rabbit anti-calbindin D-28k; Swant, product \#CB38; 1:10,000). We incubated sections for $48-72 \mathrm{~h}$ at $4^{\circ} \mathrm{C}$ with anti-CTB and anti-calbindin antibodies in $4 \%$ normal donkey serum with $0.1 \%$ Triton X-100, before washing several times in PBS at room temperature. We then incubated sections for $1 \mathrm{~h}$ at room temperature with $\mathrm{Cy} 3$-conjugated donkey anti-rabbit (Jackson ImmunoResearch Laboratories; product \#711-165$152 ; 1: 1000$ ) and Alexa488-conjugated donkey anti-goat (Jackson ImmunoResearch Laboratories; product \#705-545-147; 1:1000). After several washes in PBS, we counterstained with DAPI, mounted, and coverslipped tissue slides.

\section{Retrograde identification of CeA-PB neurons using ISH}

We injected PB of Sprague Dawley and Wistar rats with FG (see above). After 14-21 d after injection, we perfused, collected, and sectioned tissue. We used a combination of ISH and immunolabeling. We incubated free-floating CeA coronal sections $(16 \mu \mathrm{m})$ for $2 \mathrm{~h}$ at $30^{\circ} \mathrm{C}$ with rabbit anti-FG antibody (Millipore; AB153I; 1:200) in DEPC-treated phosphate buffer with $0.5 \%$ Triton X-100 supplemented with Rnasin $(40 \mu \mathrm{l} / \mu \mathrm{l}$ stock, $5 \mathrm{ml} / \mathrm{ml}$ of buffer, Promega). We rinsed sections, $(3 \times 10 \mathrm{~min})$ in DEPC-treated phosphate buffer and incubated in biotinylated donkey anti-rabbit antibody (Vector Laboratories; 1:200) for $1 \mathrm{~h}$ at $30^{\circ} \mathrm{C}$. We rinsed sections with DEPC-treated phosphate buffer and then transferred to $4 \%$ PFA. We then rinsed sections with DEPC-treated phosphate buffer, incubated for $10 \mathrm{~min}$ in phosphate buffer containing $0.5 \%$ Triton $\mathrm{X}-100$, rinsed with phosphate buffer, treated with $0.2 \mathrm{~N} \mathrm{HCl}$ for 
$10 \mathrm{~min}$, rinsed with phosphate buffer, and then acetylated in $0.25 \%$ acetic anhydride in $0.1 \mathrm{M}$ triethanolamine, $\mathrm{pH} 8.0$, for $10 \mathrm{~min}$. We rinsed sections with phosphate buffer, and postfixed with 4\% PFA for $10 \mathrm{~min}$. Before hybridization and after a final rinse with phosphate buffer, we incubated the sections in hybridization buffer for $2 \mathrm{~h}$ at $55^{\circ} \mathrm{C}$ (50\% formamide, $10 \%$ dextran sulfate, 5 $\times$ Denhardt's solution, $0.62 \mathrm{M} \mathrm{NaCl}, 50 \mathrm{~mm}$ DTT, 10 mм EDTA, 20 mм PIPES, pH 6.8, $0.2 \% \mathrm{SDS}, 250 \mathrm{mg} / \mathrm{ml}$ salmon sperm DNA, $250 \times g /$ ml tRNA). Sections were hybridized for $16 \mathrm{~h}$ at $55^{\circ} \mathrm{C}$ in hybridization buffer containing $\left[{ }^{35} \mathrm{~S}\right]$ - and [33P]- labeled singlestranded antisense CRF (nucleotides 1-1093; GenBank accession number: X03036) probes at $10^{7} \mathrm{cpm} / \mathrm{ml}$. We treated sections with $4 \mathrm{mg} / \mathrm{ml} \mathrm{RNase} \mathrm{A}$ at $37^{\circ} \mathrm{C}$ for $1 \mathrm{~h}$ and washed with $1 \times$ saline-sodium citrate, $50 \%$ formamide at $55^{\circ} \mathrm{C}$ for $1 \mathrm{~h}$ and with $0.1 \times$ saline-sodium citrate at $68^{\circ} \mathrm{C}$ for $1 \mathrm{~h}$. We then rinsed sections with phosphate buffer and incubated for $1 \mathrm{~h}$ at room temperature in avidinbiotinylated HRP (Vector Laboratories; ABC kit; 1:100). Sections were rinsed, and the peroxidase reaction was developed with $0.05 \%$ $\mathrm{DAB}$ and $0.03 \% \mathrm{H}_{2} \mathrm{O}_{2}$. We mounted sections on coated slides and dipped slides in Ilford K.5 nuclear tract emulsion (Polysciences, 1:1 dilution in double-distilled water) and exposed in the dark at $4^{\circ} \mathrm{C}$ for 4 weeks before development.

Retrograde identification and quantification of CeA-PB neurons using RNAscope

We injected $\mathrm{PB}$ of Wistar rats with FG (see above). We perfused, collected, and sectioned tissue $(14 \mu \mathrm{m})$ and mounted frozen sections directly onto Superfrost Plus slides (Thermo Fisher Scientific). We processed sections for mRNA transcripts encoding somatostatin, prodynorphin, and CRH using RNAscope, as described above. We labeled all sections for somatostatin, and alternated labeling adjacent sections for either dynorphin or CRH. According to the manufacturer's directions, we treated sections with heat, protease digestion, and hybridization of the target probes with fluorophores, Opal 520 and Opal 650 (PerkinElmer). Following hybridization of fluorophores via RNAscope, we treated all sections for FG immunoreactivity using rabbit anti-FG antibodies (Sigma Millipore; AB153-I; 1:500). We blocked sections for $30 \mathrm{~min}$ at room temperature in $2 \%$ normal donkey serum with $0.2 \%$ Triton X-100, followed by incubation in rabbit anti-FG for $24 \mathrm{~h}$ at $4^{\circ} \mathrm{C}$. We washed sections twice in PBS, followed by incubation with Cy3conjugated donkey anti-rabbit (Jackson ImmunoResearch Laboratories; \#711-165-152; 1:300) for $1 \mathrm{~h}$ at room temperature. We washed sections twice in PBS, counterstained with DAPI, and coverslipped with ProLong Gold mounting medium (Invitrogen). Each batch included sections processed with negative and positive control probes to ensure specificity of the target probes.

Following RNAscope and FG immunolabeling, we quantified the neurochemical phenotype of CeA-PB neurons by observing and photographing sections with a Leica Microsystems TCS SP8 confocal microscope. We held excitation and detection parameters constant across all sections. We imaged full sections using a $5 \times$ objective, and CeA and surrounding structures using $20 \times$ oil immersion objective. We reconstructed sections using Leica LAS X Navigator tiling software. Finally, we analyzed sections using Neurolucida (MBF Bioscience), thresholding the FG signal to reduce nonspecific immunolabelling, and manually drawing boundaries for CeA (Paxinos and Watson, 2013). We hindpaw $(\boldsymbol{H})$ are higher.
B

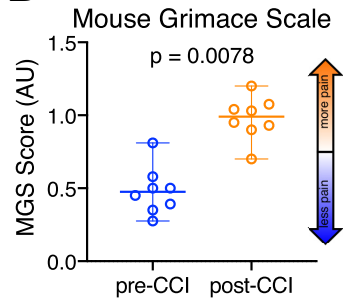

E
D

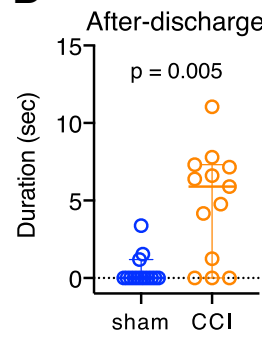

G

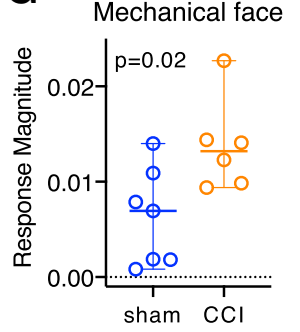

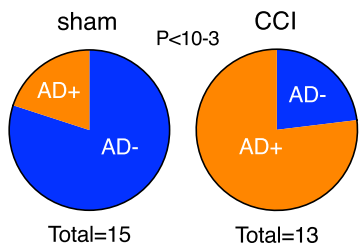

H

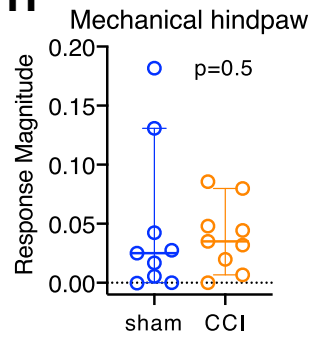

Figure 1. C(I-ION causes behavioral signs of pain and amplification of neuronal activity in the PB nucleus. $\boldsymbol{A}-\boldsymbol{H}$, All data

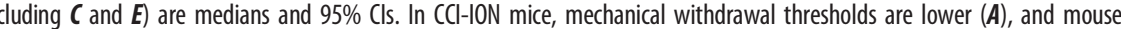
ron spontaneous firing rates are higher $(\boldsymbol{F})$, and magnitudes of responses to mechanical stimuli applied to the face $(\boldsymbol{G})$ and

manually identified and quantified FG-positive neurons, followed by identifying those neurons that colabeled for somatostatin, dynorphin, and $\mathrm{CRH}$. We collected every fifth section through CeA and counted every neuron within CeA. We included only sections with at least 20 FG-positive neurons to control for variations in sectioning angle, edge effects, and deposition or uptake of FG at the injection site. From each of the 5 animals analyzed, we included $\sim 20$ sections (see Results) for this quantification. We compared distributions of somatostatin-labeled FG-positive neurons between the two assays (i.e., RNAscope for somatostatin and dynorphin vs somatostatin and CRH) and found no differences.

\section{Statistical analysis}

We analyzed group data using GraphPad Prism version 8 for Mac (GraphPad Software). Data are presented, unless otherwise noted, as median values $\pm 95 \%$ CIs. We used nonparametric Mann-Whitney $U$ tests, Wilcoxon matched-pairs signed rank tests, Friedman's repeated-measures tests, and a binomial test, as noted below (see Results). We used nonparametric methods because they typically represent a more cautious approach, especially for smaller samples, and because they remove the burden of assumptions about the distribution (Krzywinski and Altman, 2014).

\section{Results}

\section{CCI-ION results in signs of pain}

As we and others described previously (Bennett and Xie, 1988; Akintola et al., 2017), CCI-ION resulted in pain-like behaviors in 
A
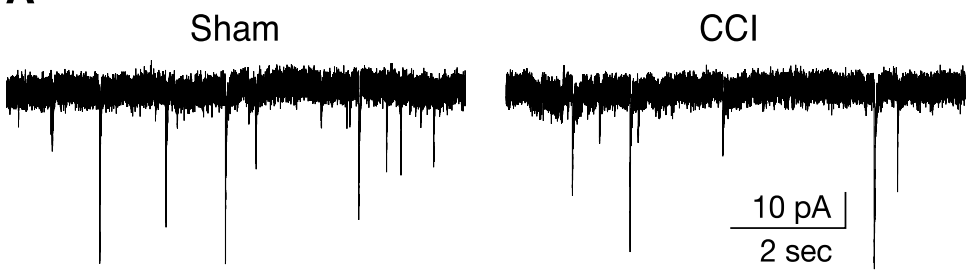

B

mIPSCs frequency

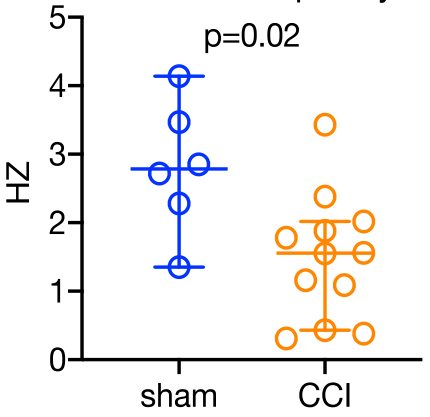

C

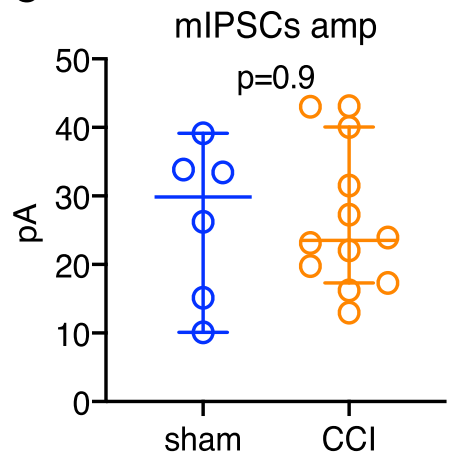

D

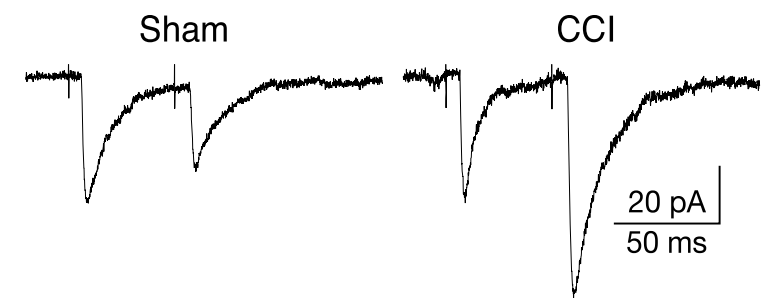

E

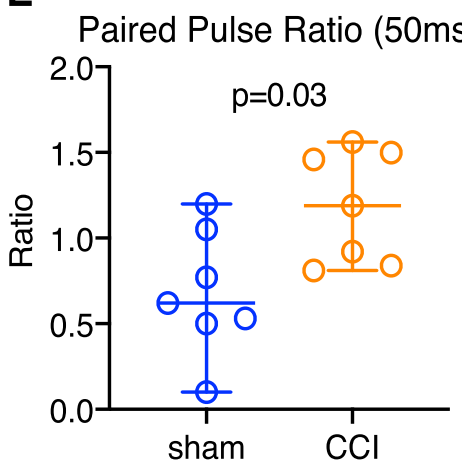

$\mathbf{F}$

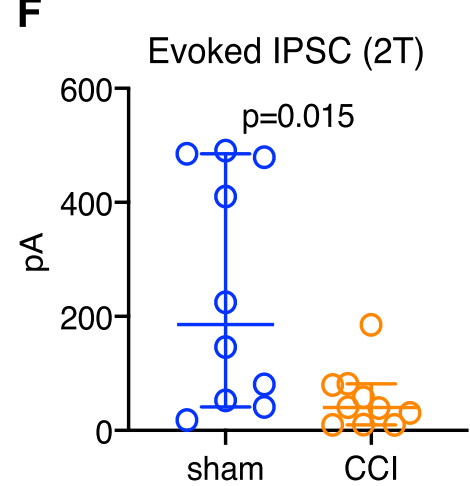

Figure 2. CCl-ION attenuates inhibitory inputs to the LPB from the CeA. Data are medians and $95 \% \mathrm{Cls}$. $A$, Example recordings of mIPSCs from PB neurons from sham and CCI mice. $\boldsymbol{B}$, mIPSC frequency is lower in $C \mathrm{CI}-\mathrm{ION}$ mice, whereas their amplitudes remain indistinguishable from controls (C). $\boldsymbol{D}$, Example traces of IPSCs evoked in LPB neurons by optogenetically activating inhibitory CeA afferents with pairs of light stimuli. $\boldsymbol{E}$, PPRs $(50 \mathrm{~ms})$ are higher, consistent with a reduction in synaptic efficacy. $\boldsymbol{F}$, Amplitude of IPSC evoked by stimulating CeA afferents at twice stimulus threshold are lower in CCI-ION mice.

mice, assessed 3 weeks after surgery. Thresholds for withdrawal from mechanical stimuli in CCI-ION mice were more than twofold lower (median of $1.2 \mathrm{~g}$; 0.3-2.2 $\mathrm{g} 95 \% \mathrm{CI}$ ) compared with shams (2.8 $g$; 1.8-4.2 $g$ 95\% CI; $p=0.009$, Mann-Whitney $U=2$; Fig. 1A). This indicates that CCI-ION resulted in persistent hyperalgesia.

We have previously reported that the mouse grimace scale is a reliable and sensitive metric for the assessment of ongoing pain in mice with CCI-ION (Akintola et al., 2017). Consistent with this, CCI-ION mice had grimace scale scores that were more than twofold higher than those in sham mice (median $=0.99$,
0.70-1.20 95\% CI vs $0.475,0.275-0.810$ 95\% CI; $p=0.0003$, Mann-Whitney $U=1$; Fig. $1 B)$.

These findings indicate that mice with CCI-ION exhibited signs of both ongoing ("spontaneous") pain as well as mechanical hypersensitivity.

\section{PB neuron activity is amplified after CCI-ION}

We have previously demonstrated that $\mathrm{PB}$ activity is amplified in rats with chronic pain, induced by CCI-ION (Uddin et al., 2018). Here we demonstrate that this pathologic phenomenon occurs also in mice. We also show that, as in the rat, CCI-ION is associated with amplified responses to stimuli applied both to the face and to the hindlimb.

Figure $1 C$ depicts representative extracellular spikes recorded from isolated PB neurons from an anesthetized, sham-operated animal (Fig. $1 C$, top) and from a CCI-ION mouse (Fig. 1C, bottom). Activity evoked in response to face pinch, applied through calibrated forceps, was eightfold higher in the CCI-ION neuron, compared with the sham. Significantly, the PB neuron from the CCI-ION mouse showed pronounced $\mathrm{ADs}$, responses that far outlasted the stimuli, which were of longer duration and frequency compared with those in the control neuron. In this neuron, ADs lasted $>40 \mathrm{~s}$.

PB neurons recorded from CCIION mice were 4 times as likely to exhibit ADs, with $77 \%$ of tactile-responsive neurons from CCI animals and $20 \%$ of neurons from sham animals displaying $\mathrm{ADs}\left(p<10^{-3}\right.$, binomial test; Fig. $1 E)$. AD duration was longer in CCI-ION mice $(n=13$, median $=$ $5.9 \mathrm{~s}, 95 \% \mathrm{CI}=0-7.3 \mathrm{~s}$ ) than in shamoperated mice $(n=14$, median $=0 \mathrm{~s}$, 95\% CI =0-1.2; Mann-Whitney $U=$ 27.50, $p=0.005$; Cohen's $d=1.7$, "large" effect size; Fig. 1D).

We quantified responses of $\mathrm{PB}$ neurons during noxious application of calibrated forceps to the face or hindpaws. Neuronal response magnitudes are expressed as arbitrary units, computed as frequency (in $\mathrm{Hz}$ ) divided by stimulus magnitude (integral of force applied). The magnitude of response to stimulation of the face, but not the hindpaw, was higher in CCI-ION animals, compared with controls (Fig. 1G, H). Neurons recorded from mice with CCI responded to facial stimuli with a median magnitude of 0.013 units $(95 \% \mathrm{CI}=0.009-0.023, n=6)$, whereas neurons recorded from sham-injured animals responded with a median magnitude of 0.007 units (95\% CI $=0.001-0.014, n=7$; Mann-Whitney $U=5$; $p=0.02$; Fig. $1 G$; Cohen's $d=1.5$, "large" effect size). In 

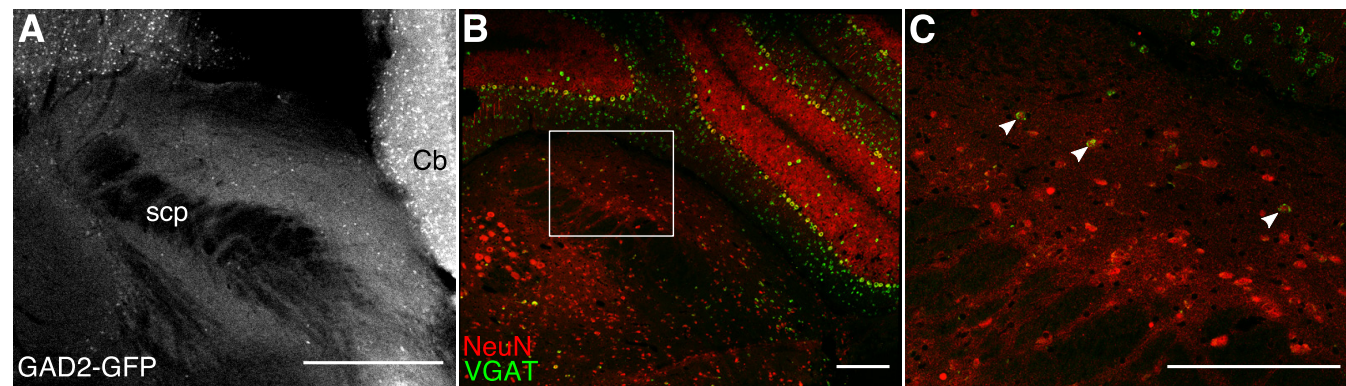

Figure 3. $P B$ nuclei contain few inhibitory, GABAergic interneurons. $A$, A representative image of coronal sections from GAD2-GFP mice represents few GABAergic neurons (bright white puncta) in PB. B, C, Few LPB neurons express VGAT. Representative images of coronal sections through the PB of Wistar rats showing immunoreactivity for NeuN (red) and expression of VGAT mRNA (green). Square inset, A portion of LPB enlarged in C. White arrows indicate example VGAT neurons. Scale bars, $250 \mu \mathrm{m}$.

contrast, neurons from both conditions responded similarly to hindpaw stimuli; sham animals $(n=9)$ had a median response magnitude of 0.025 units ( $95 \% \mathrm{CI}=0.000-0.131)$, and CCI-ION cells had a median response of 0.035 units $(95 \% \mathrm{CI}=0.007$ 0.080; Mann-Whitney $U=32.5, p=0.5$; Fig. $1 H$ ).

Spontaneous firing rates of $\mathrm{PB}$ neurons in CCI-ION mice were higher than those in control animals. Neurons from CCI-ION mice $(n=9)$ had a median firing rate of $13.9 \mathrm{~Hz}(95 \% \mathrm{CI}=1.7-19.0 \mathrm{~Hz})$, whereas neurons from sham animals $(n=9)$ had a median firing rate of $3.5 \mathrm{~Hz}(95 \% \mathrm{CI}=0.8-7.7 \mathrm{~Hz}$; Mann-Whitney $U=$ 16, $p=0.03$; Fig. 1F; Cohen's $d=1.4$, "large" effect size).

These data indicate that, as we previously reported in rats (Uddin et al., 2018), CCI-ION results in amplification of PB neuronal activity. In mice, CCI-ION manifested as an increase in spontaneous firing rates and in larger responses to mechanical stimuli. Most prominent was the increase in the magnitude of ADs in animals with pain after CCI.

\section{Inhibitory inputs to $\mathrm{PB}$ are reduced after CCI-ION}

We and others have previously shown that amplified neuronal activity in chronic pain can result from maladaptive disinhibition of CNS structures (for review, see Masri and Keller, 2012; Prescott, 2015). To test whether disinhibition is associated with the amplified activity of $\mathrm{PB}$ neurons after CCI-ION, we recorded from $\mathrm{PB}$ neurons in acute slices. Figure $2 \mathrm{~A}$ depicts traces of whole-cell recordings from $\mathrm{PB}$ neurons from CCI-ION and control mice. mIPSCs appear as inward currents because of the high $\left[\mathrm{Cl}^{2-}\right]$ in the patch pipette (see Materials and Methods). The frequency of these events is $\sim 50 \%$ lower in the example from the CCI-ION animal, compared with the control. For group comparisons, we first averaged data from all neurons recorded from a particular mouse and used these averages as our individual samples (i.e., sample size $=$ number of mice). This analysis revealed that the frequency of mIPSCs in CCI-ION mice was approximately half that in controls (Cohen's $d=1.4$ ). Median mIPSC frequency in PB cells from CCI-ION mice $(n=12)$ was $1.6 \mathrm{~Hz}(95 \%$ CI: $0.4-2.0 \mathrm{~Hz})$, compared with $2.8 \mathrm{~Hz}(95 \%$ $\mathrm{CI}=1.4-4.1$; Mann-Whitney $U=11, p=0.02$; Fig. $2 B)$ in sham animals $(n=6)$. In contrast, the amplitudes of mIPSCs in CCIION mice were indistinguishable from that in controls. Neurons from CCI-ION mice ( $n=12$ mice) had a median mIPSC amplitude of $23.5 \mathrm{pA}(95 \% \mathrm{CI}=17.3-40.0 \mathrm{pA})$, and neurons from sham animals $(n=6)$ had a median amplitude of $29.8 \mathrm{pA}(95 \%$ $\mathrm{CI}=10.1-39.1$ pA; Mann-Whitney $U=35, p=0.9$; Fig. $2 C$ ).

Changes in the amplitudes of synaptic potentials might be masked by parallel changes in input resistance of the recorded neurons. However, there was no difference $(p=0.9$; MannWhitney $U=225$ ) in resistance of neurons from CCI-ION mice
( median $=771 \mathrm{M} \Omega ; 95 \% \mathrm{CI}=631-983 \mathrm{M} \Omega$ ), compared with controls $($ median $=691 \mathrm{M} \Omega ; 95 \% \mathrm{CI}=617-1056 \mathrm{M} \Omega$ ). In currentclamp recordings, there was no difference $(p=0.19$; MannWhitney $U=176)$ in resting membrane potential of neurons from CCI-ION mice (median $=-68 \mathrm{mV} ; 95 \% \mathrm{CI}=-69$ to -67 ), compared with controls (median $=-69 \mathrm{mV} ; 95 \% \mathrm{CI}=$ -70 to $-67 \mathrm{mV})$.

\section{Source of inhibition}

$\mathrm{PB}$ is reported to contain only a small number of inhibitory neurons (Guthmann et al., 1998; Yokota et al., 2007). Consistent with these findings, examination of sections through $\mathrm{PB}$ of GAD2-GFP mice revealed that PB, and the LPB nucleus in particular, contain only a small number of GAD-GFP neurons (Fig. $3 A$ ). In contrast, other regions, such as the cerebellum, contain a high density of these neurons.

Similar to mice, sections through the PB of Wistar rats reveal a relatively a small proportion of $\mathrm{PB}$ neurons express VGAT (Fig. $3 B, C$ ). The lateral and ventral portions of the $\mathrm{PB}$ contain the densest collection of these GABAergic neurons, and a small number of isolated VGAT neurons appear throughout the PB. We estimated the proportion of GABAergic LPB neurons by quantifying the number of NeuN immuno-positive cells that express VGAT mRNA in nonadjacent coronal sections (see Materials and Methods). We calculated the proportion for each individual section, sampled at $280 \mu \mathrm{m}$ intervals from throughout the rostro-caudal extent of LPB. The median percentage of VGAT neurons was $12 \%(95 \% \mathrm{CI}=9-15 \% ; n=8$ sections, 1 male and 1 female rat). The relative paucity of GABAergic neurons in $\mathrm{LPB}$ suggests that major inhibitory inputs to $\mathrm{PB}$ are extrinsic to this nuclear complex.

\section{Mouse anatomy}

Retrograde tracing

To identify the inhibitory inputs that might be involved in disinhibition of PB following CCI-ION, we used retrograde and anterograde anatomic tracing, in both the rat and mouse. Injection of a retrograde neuronal tracer, $\mathrm{CTB}$, into the right PB of GAD2GFP mice $(n=3)$ revealed a high density of retrogradely labeled neurons in the ipsilateral CeA. We found lower density of retrograde labeling in the zona incerta and subthalamic nucleus, in the paraventricular and lateral hypothalamus, and in the insular cortex, all ipsilateral to the injection site. Figure $4 A-C$ shows coronal sections through the $\mathrm{CeA}$, demonstrating that retrogradely labeled neurons occupy much of the central nucleus.

Because CeA is thought to be composed almost exclusively of GABAergic neurons (Duvarci and Pare, 2014), these findings indicate that inhibitory neurons in the mouse CeA provide dense 

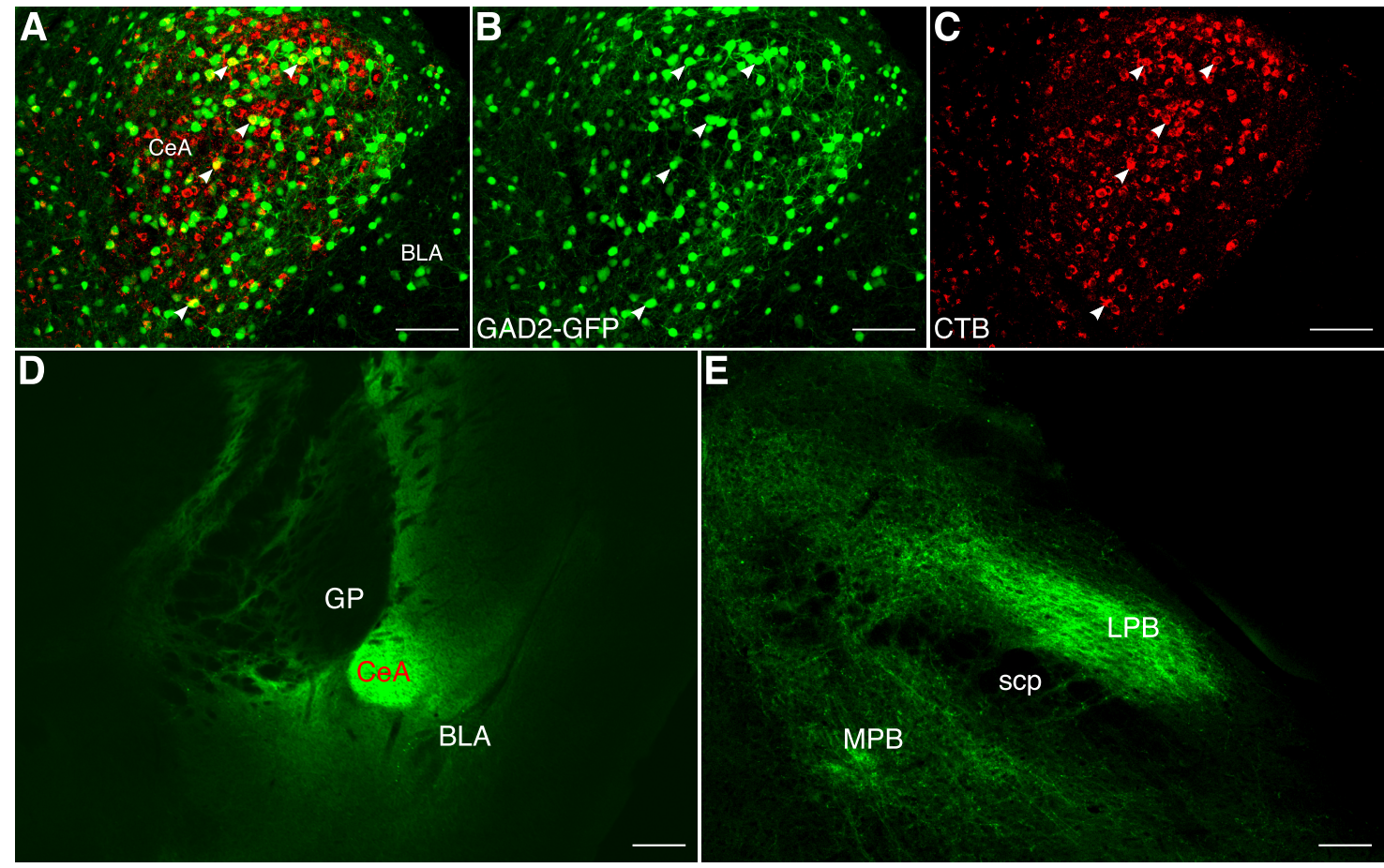

Figure 4. Retrograde and anterograde tracing in mice reveals that CeA provides dense inhibitory input to PB. $A-C$, Representative images of coronal sections through the CeA of GAD2-GFP mice that received injections of CTB in PB. Merged signals $(\boldsymbol{A})$ from GAD2-GFP $(\boldsymbol{B})$ and CTB $(\boldsymbol{C})$ reveal CeA-PB projection neurons (white arrows). $\boldsymbol{D}, \boldsymbol{E}$, Representative images of coronal sections through (eA and LPB, respectively, from GAD2-Cre mice that received injection of a (re-dependent viral construct (AAV-DI0-hChR2-eYFP) into CeA. Abundant axons expressing eYFP can be seen in LPB (E). Scale bars: $A-C, 100 \mu \mathrm{m} ; \boldsymbol{D}, \boldsymbol{E}, 250 \mu \mathrm{m}$.

inhibitory inputs to PB. Consistent with this conclusion, many neurons in CeA that were retrogradely labeled following CTB injection in $\mathrm{PB}$ were GAD2-positive (Fig. $4 A-C$, arrows). We further characterize the chemical identity of these CeA-LPB neurons below.

\section{Anterograde tracing}

We confirmed the existence of an CeA-LPB pathway using an anterograde tracing approach. We injected into the right CeA of GAD-Cre mice $(n=3)$ the viral construct, AAV-EF1a-DIOhChR2-EYFP (this construct was used for optogenetic manipulation studies described below). Images of sections through the injection site demonstrate that EYFP expression is restricted primarily to CeA (Fig. $4 D$ ). Sections through $\mathrm{PB}$ reveal a high density of labeled axons in PB (Fig. 4E). These projections from the CeA were notably dense in the lateral regions of $\mathrm{PB}$, ipsilateral to the injection site. These findings confirm the existence of a dense CeA-LPB inhibitory pathway in the mouse.

\section{Rat anatomy}

Retrograde tracing

To determine whether a similar CeA-LPB pathway exists in the rat we first used the retrograde neuronal tracer, CTB. Figure $5 \mathrm{~A}$ depicts a coronal section through $\mathrm{PB}$ of an adult rat injected with $\mathrm{CTB}$, revealing an injection site covering much of the $\mathrm{PB}$ region. We made similar injections in the right PB of 6 adult male Sprague Dawley rats. These injections resulted in dense, retrograde labeling of somata in the ipsilateral CeA (Fig. 5B,C). Similar to the mouse, we found a lower density of retrogradely labeled neurons in the zona incerta, the paraventricular and lateral hypothalamus, and the insular cortex, ipsilateral to the injection.
To determine the phenotype of these retrogradely labeled neurons, we double-labeled the sections with antibodies for markers of GABAergic neurons (see Materials and Methods). Figure $5 B-F$ shows examples of these retrogradely labeled neurons immunoreactive for the calcium binding protein, calbindin. A large proportion of CTB-positive CeA neurons did not label for calbindin (Fig. $5 C, D$ ), suggesting that they represent other classes of GABA neurons, a possibility we explore below. These data suggest that, like in the mouse, the CeA-LPB pathway in the rat consists of a dense projection of a heterogeneous population of GABAergic CeA neurons.

We confirmed these findings using a different retrograde tracer, FG. By making discrete iontophoretic injections of FG, we were better able to restrict our injections to those portions of the PB that have been previously implicated in processing and relaying nociceptive information (Cechetto et al., 1985; Roeder et al., 2016; Chiang et al., 2019). Further, while the CTB experiments in the rat were performed in male Sprague Dawley rats, FG tracing was done in Sprague Dawley and Wistar rats of both sexes. We observed no difference in the pattern of CeA-LPB projection between male and female animals, nor between the two strains of rat.

Figure 5 shows coronal sections through $\mathrm{PB}$, demonstrating injection sites revealed with reflected (Fig. $5 G$ ) and transmitted light (Fig. 5I) microscopy. In contrast to our CTB injections above, injections of FG were restricted primarily to the LPB. We observed strong deposition of FG throughout the LPB, with little dissipation into the medial $\mathrm{PB}$ or other nearby nuclei, such as the Köllicker-Fuse or the locus ceruleus. We made similar injections into the right LPB of Sprague Dawley rats ( $n=3$ of each sex) and bilaterally into the LPB of Wistar rats ( $n=3$ of each sex).

Sections through the amygdala reveal a pattern of labeled somata similar to that observed after CTB injections (Fig. $5 \mathrm{H}, \mathrm{J}$ ). 

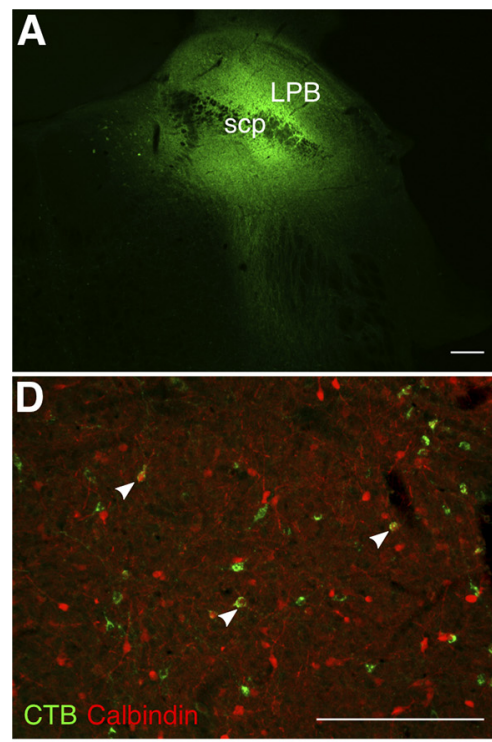

$\mathbf{B}$
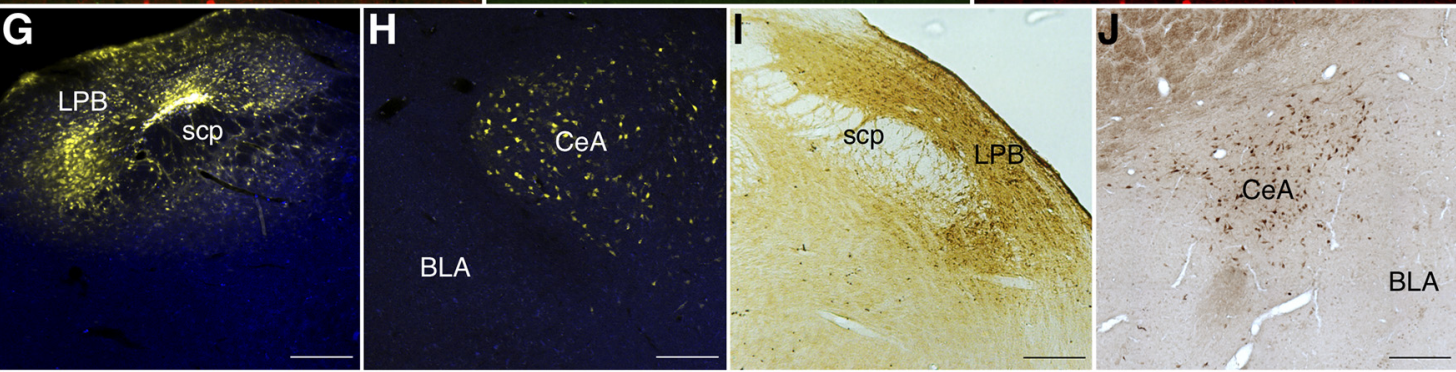

$\mathbf{K}$

E

E

$\mathrm{CeA}$
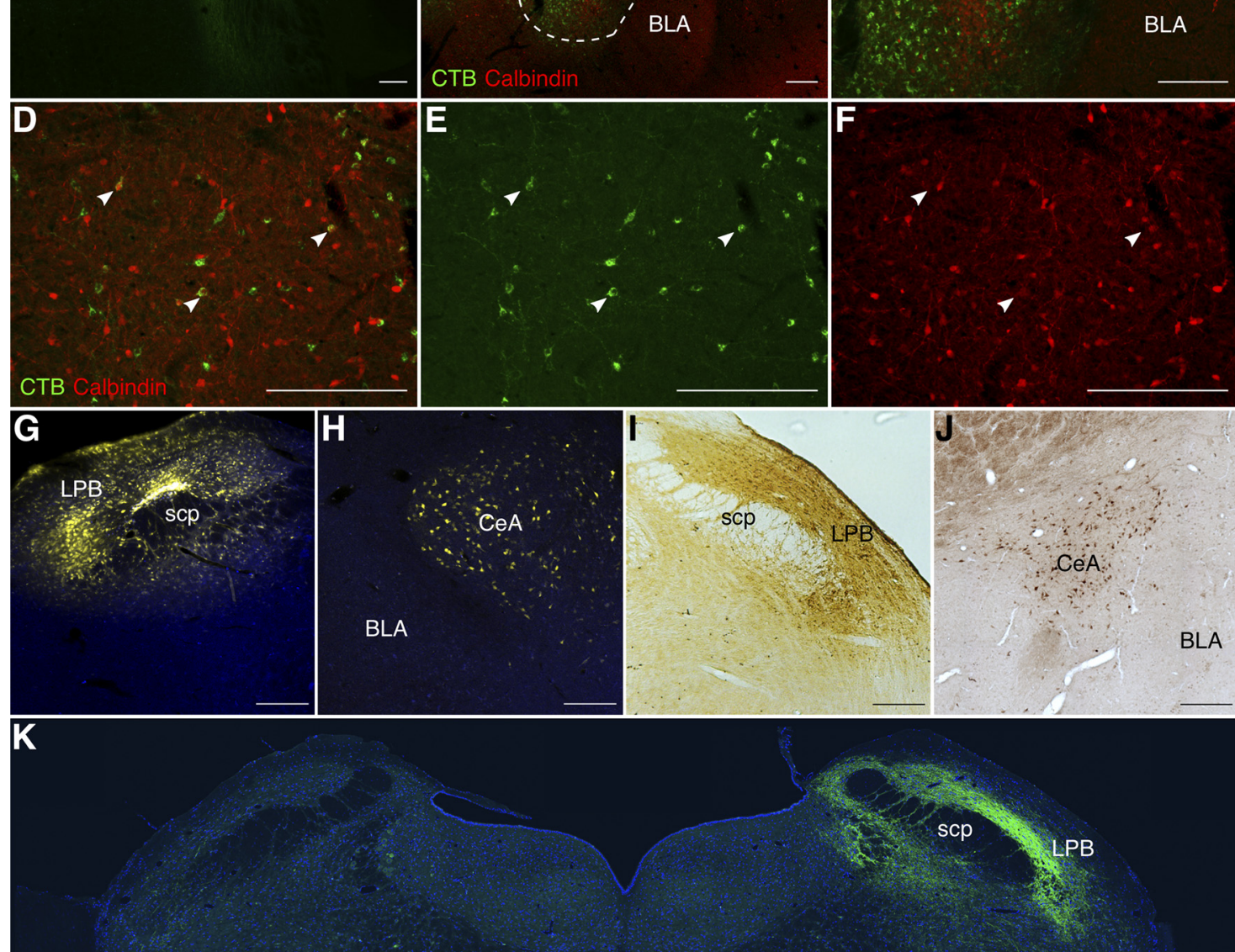

L

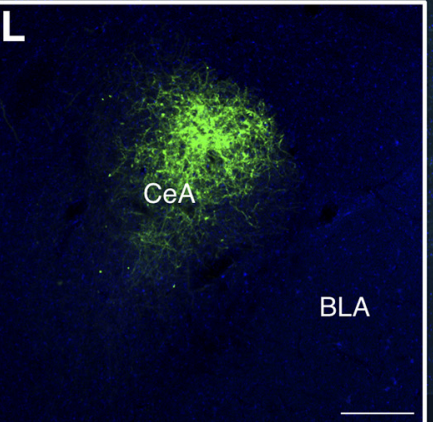

Figure 5. Retrograde and anterograde tracing in rats reveals that CeA provides robust inhibitory input to PB. $A-F$, Representative images of coronal sections through $P B$ and CeA of rats that received injections of (TB. $A$, Dense deposition of (TB (immunoreactivity in green) at the injection site in PB. $\boldsymbol{B}, \boldsymbol{C}$, Numerous retrogradely labeled neurons are seen in CeA in low- and high-magnification images of merged immunoreactivity signals from (TB (green) and calbindin (red). High-magnification image of merged (D), CTB (E), and calbindin $(\boldsymbol{F})$ signals reveals sparse colabeling of individual neurons (white arrows). $\mathbf{G}-\boldsymbol{J}$, Representative images of coronal sections through PB and CeA of rats that received discrete iontophoretic injections of FG into PB. Restricting FG depositions primarily to the LPB $(G, I)$ revealed that the densest input to LPB arises from CeA $(\boldsymbol{H}, \boldsymbol{J})$. FG depositions and labeled neurons were identified by their fluorescent signal $(\boldsymbol{G}, \boldsymbol{H}$, yellow) and immunoreactivity $(\boldsymbol{I}, \boldsymbol{J}$, brown reaction product). $\boldsymbol{K}, \boldsymbol{L}$, Representative images of coronal sections through $L P B$ and CeA, respectively, of CRH-Cre rats that received injection of a Cre-dependent viral construct (AAV-DIO-hChR2-eYFP) into CeA. Scale bars, $250 \mu \mathrm{m}$. 
Neurons retrogradely labeled with FG were distributed throughout the ipsilateral CeA, but did not extend into the basolateral or medial nuclei of the amygdala. Below we describe our quantification of these projections, and identification of their chemical phenotypes. Consistent with our injections of CTB, we observed a small number of $\mathrm{FG}^{+}$cells in the paraventricular and lateral hypothalamus, in the zona incerta, and insular cortex, all ipsilateral to the injection site. We observed no differences in either the pattern of distribution or laterality of these retrogradely identified cells between sex or animal strain.

\section{Anterograde tracing}

We confirmed the retrograde data in the rat with the use of anterograde viral tracing. We expanded the study by using a transgenic rat that expresses Cre exclusively in $\mathrm{CRH}$ neurons (Pomrenze et al., 2015). This allowed us to restrict the expression of the viral constructs to $\mathrm{CeA}$, as the expression of $\mathrm{CRH}$ in the amygdala is limited to this structure (Pomrenze et al., 2015) (Figs. 5, 6).

Injection of the viral construct AAV-DIO-hChR2-EYFP into the CeA of CRH-Cre rats ( $n=2$ of each sex) resulted in highly localized expression within the CeA (Fig. $5 L$ ). These injections resulted in dense labeling of axons in the $\mathrm{PB}$, primarily in ipsilateral LPB, but also included MPB (Fig. $5 K$ ). We observed no difference in the laterality or the distribution of projections between male and female rats.

These data suggest that the CeA-LPB pathway in the rat includes a dense projection from inhibitory neurons in the CeA to the LPB. Given the evidence from retrograde and anterograde anatomic approaches in both rats and mice, CeA is well positioned to provide a robust source of inhibitory control over nociceptive information processing in the LPB.

\section{CCI-Pain results in suppressed CeA-PB inputs}

The dense GABAergic, inhibitory projection from CeA to $\mathrm{PB}$ suggests that this pathway is involved in the reduced inhibition of PB neurons after CCI-ION. We tested this hypothesis by comparing CeA-LPB inputs in control and CCI-ION mice. In live slices through $\mathrm{PB}$, we recorded IPSCs from LPB neurons, evoked by optogenetic stimulation of CeA afferents to $\mathrm{PB}$ (Fig. $2 D-F$ ). We accomplished this by expressing ChR2 selectively in GABAergic neurons, by injecting Cre-dependent viral constructs in CeA of GAD2-CRE mice (see Source of inhibition).

Brief $(0.3 \mathrm{~ms})$ pulses of light-evoked robust IPSCs in LPB neurons (Fig. 2D). To compare the efficacy of these evoked IPSCs in different neurons, we evoked pairs of stimuli, separated by $50 \mathrm{~ms}$, and computed the paired-pulse ratio (PPR), where a higher ratio is associated with "weaker" synapses, and a lower ratio with "stronger" synapses (Zucker, 1989; Kim and Alger, 2001; Sanabria et al., 2004). Because PPR is sensitive to stimulus intensity, we calibrated the optical stimulus for each neuron, by varying laser intensity, to obtain a response that was at twice the threshold stimulus intensity. For each neuron, we calculated PPR as the mean of the second response divided by the mean of the first (Kim and Alger, 2001), and then averaged the PPRs computed for all neurons recorded from the same animal. These averages were then used for statistical comparisons.

PPR recorded from neurons in CCI mice was nearly twice as large compared with that in control animals. Neurons from CCIION mice ( $n=7$ mice) had a median PPR of 1.19 (95\% CI $=0.81-$ $1.56)$, whereas neurons from sham animals $(n=7)$ had a median
PPR of 0.62 (95\% CI =0.10-1.20; Mann-Whitney $U=7, p=0.03$; Fig. $2 E$; Cohen's $d=1.4$ ).

The amplitudes of the first IPSCs, evoked at twice stimulus threshold, was nearly fivefold smaller in CCI-ION mice, compared with controls (Cohen's $d=1.3$ ). Neurons from CCI-ION mice ( $n=10$ mice) had a median evoked IPSC amplitude of $40.1 \mathrm{pA}$ (95\% CI=9.9-81.8 pA), whereas neurons from sham animals $(n=10)$ had a median amplitude of $185.6 \mathrm{pA}(95 \%$ $\mathrm{CI}=41.2-482.2$ pA; Mann-Whitney $U=18, p=0.015$; Fig. $2 F$ ).

These data suggest that inhibitory inputs from CeA to LPB are reduced in animals with chronic pain, compared with sham-operated controls. The reductions in mIPSC frequency and evoked IPSC amplitude, the increase in PPR, with no change in mIPSC amplitude, all point to presynaptic changes in this CeA-LPB circuitry. These electrophysiological data, coupled with our anatomic evidence, suggest that the amygdalo-PB pathway may play a causal role in the development of neuropathic pain after CCI.

\section{Neurochemical identity of CeA-PB neurons}

To further characterize the neurochemical phenotype of CeA$\mathrm{PB}$ neurons, we used a combination of ISH and RNAscope. These revealed that CeA-LPB neurons are a heterogeneous population, including neurons expressing $\mathrm{CRH}$, somatostatin, and dynorphin; a subset of these CeA-LPB neurons expressed both somatostatin and dynorphin mRNA, or somatostatin and CRH mRNA.

Figure $5 G, I$ show coronal sections through the PB of FGinjected rats. Only animals in which the injection site was restricted to LPB were used for further processing via ISH $(n=2$ Sprague Dawley rats and 3 Wistar rats) or RNAscope $(n=2$ female, 3 male Wistar rats).

Figure $6 A, B$ depict coronal sections through CeA of FGinjected rats that were processed for ISH and visualized with a combination of bright-field and epifluorescence microscopy. FG-positive neurons occupy much of the CeA. FG-positive neurons expressing mRNA for either $\mathrm{CRH}$ or dynorphin are depicted in Figures $6 A$ and $6 B$, respectively. A small proportion of CeA-LPB neurons expressed CRH, whereas a substantial proportion of the FG-labeled-positive population of CeA neurons was positive for dynorphin mRNA expression.

Figure $6 C, D$ shows coronal sections through the CeA of FGinjected rats that were processed using RNAscope, and visualized with confocal microscopy. FG-positive neurons appear white, those expressing mRNA for somatostatin are green, and $\mathrm{CRH}$ or dynorphin mRNA-positive neurons are red (Fig. $6 C$ and Fig. $6 D$, respectively). Not only did CeA FG-positive neurons express mRNA associated with one of these phenotypes, a substantial subpopulation coexpressed mRNA for somatostatin and either $\mathrm{CRH}$ or dynorphin.

We quantified the number of retrogradely labeled CeA-LPB neurons displaying these phenotypes. Figure $6 E, F$ depicts the fraction of CeA FG-positive neurons that expressed only one phenotype, that were double-labeled for two phenotypes, as well as the total fraction of identified versus unidentified FG-positive neurons in each assay, as mean and 95\% CIs. Separate sections were treated with primers either for somatostatin and dynorphin $(n=23$ sections, 5 animals; Fig. $6 E$ ), or somatostatin and $\mathrm{CRH}$ $(n=21$ sections, 5 animals; Fig. $6 F)$. We included in these analyses only those sections that contained a minimum of 20 FG-positive neurons, to control for variation in the intensity of FG deposition and uptake at the injection site. Dynorphin-expressing neurons accounted for the largest fraction of these 


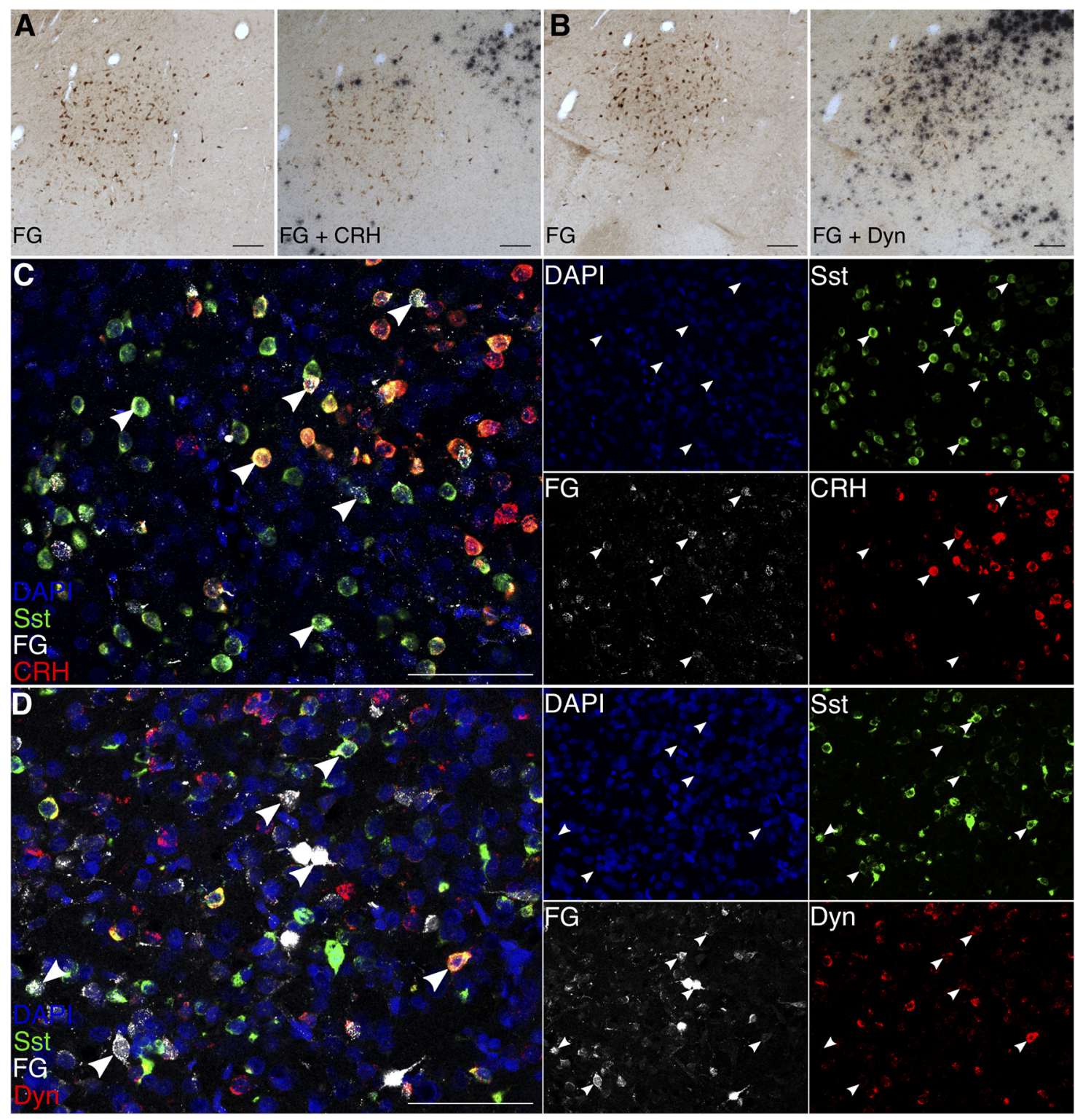

$\mathbf{E}$

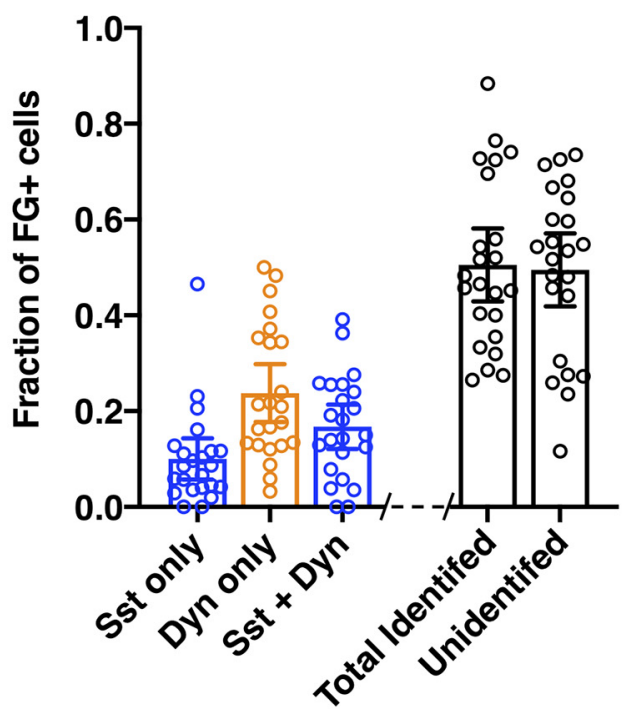

$\mathbf{F}$

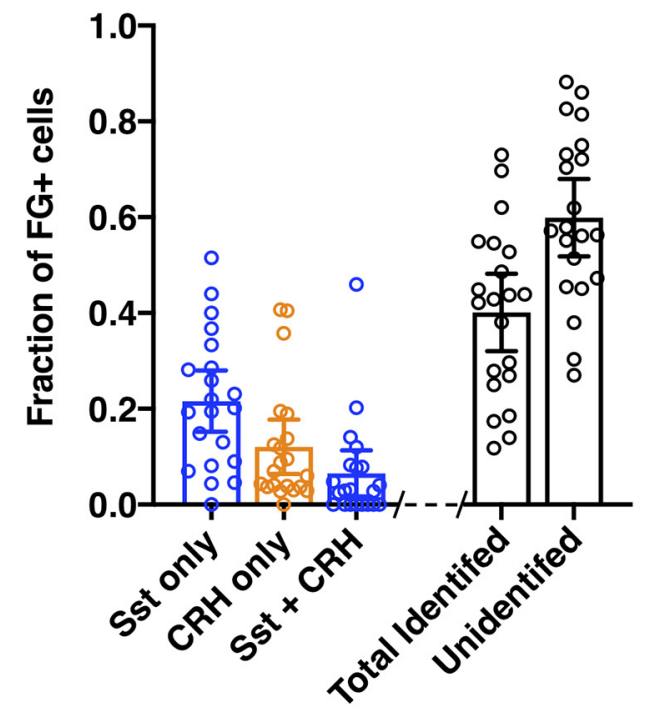

Figure 6. Neurochemical identification of CeA-LPB neurons reveals a heterogeneous population expressing dynorphin, somatostatin, and CRH mRNAs. $A, B$, Representative images of coronal sections through CeA reveal limited expression of CRH mRNA $(\boldsymbol{A})$ but robust expression of dynorphin mRNA $(\boldsymbol{B})$ in CeA-LPB neurons. Left panels, FG-immunoreactive cells (brown reaction product) before deposition of silver grains via ISH. Right panels, Same section after labeling for (RH or dynorphin mRNA (black reaction product), in $\boldsymbol{A}$ and $\boldsymbol{B}$, respectively. C, D, Representative 
phenotypes. The mean fraction of FG-positive CeA-LPB neurons that express dynorphin only was $0.24(95 \% \mathrm{CI}=0.18-0.30)$, compared with a mean of 0.10 for somatostatin only $(95 \% \mathrm{CI}=0.06-$ 0.14 ) and a mean of 0.17 for dynorphin and somatostatin $(95 \%$ $\mathrm{CI}=0.12--0.21$; Fig. $6 E$ ). The total mean fractions of CeA-LPB neurons expressing dynorphin or somatostatin were 0.41 ( $95 \%$ $\mathrm{CI}=0.33-0.48)$ and $0.27(95 \% \mathrm{CI}=0.20-0.33)$, respectively. The mean fraction of FG-positive neurons expressing $\mathrm{CRH}$ only was $0.12(95 \% \mathrm{CI}=0.06-0.18)$, compared with a mean of 0.22 for somatostatin only $(95 \% \mathrm{CI}=0.15-0.28)$ and a mean of 0.06 for CRH and somatostatin $(95 \% \mathrm{CI}=0.02-0.11$; Fig. $6 F$ ). The total mean fractions of CeA-LPB neurons expressing CRH or somatostatin were $0.18(95 \% \mathrm{CI}=0.10-0.27)$ and $0.28(95 \% \mathrm{CI}=0.21-$ $0.35)$, respectively.

These findings indicate that, of neurons comprising the inhibitory CeA-LPB, almost half express dynorphin, and many of these neurons coexpress somatostatin. A smaller population of neurons in this pathway express somatostatin alone and/or $\mathrm{CRH}$. We did not probe for other neurochemical markers of CeA neurons, such as protein kinase C- $\delta$ or neurotensin (Torruella-Suárez et al., 2019; Wilson et al., 2019).

\section{Causality}

To determine whether the CeA-PB pathway plays a causal role in the regulation of pain sensation and the development of chronic pain, we tested the prediction that manipulating the CeA-PB pathway, pharmacologically or optogenetically, would result in changes in behavioral metrics of pain. Consistent with a causal role in chronic pain for this pathway, disinhibiting the $\mathrm{PB}$ caused increases in pain metrics, whereas inhibiting the $\mathrm{PB}$ alleviated signs of chronic pain; activating the CeA-LPB pathway reduced metrics of acute pain.

\section{Pharmacological inhibition of $P B$}

We first tested the prediction that inhibition of $\mathrm{PB}$ will suppress pain metrics in animals with CCI-Pain. To inhibit PB, we infused muscimol, the $\mathrm{GABA}_{\mathrm{A}}$ receptor agonist, or a saline-vehicle control into the LPB of CCI-ION injured rats (see Materials and Methods).

Figure $7 B, C$ depicts the facial mechanical withdrawal thresholds for individual animals, as well as the median and 95\% CIs for each time point. Ipsilateral to the injury, left face thresholds changed across time points (Friedman's $F=10.22 ; p=0.0065$; Fig. $7 B)$, from a median of $34.8 \mathrm{~g}$ at baseline $(95 \% \mathrm{CI}=29.9-$ $37.0 \mathrm{~g})$ to $20.47 \mathrm{~g}$ after CCI $(95 \% \mathrm{CI}=14.9-30.3 \mathrm{~g}), 24.9 \mathrm{~g}$ after saline (95\% CI $=13.3-32.5 \mathrm{~g}$ ), and $34.8 \mathrm{~g}$ after muscimol infusion $(95 \% \mathrm{CI}=25.4-47.5 \mathrm{~g})$. Individual comparisons (after Dunn's correction) revealed a difference (at $\alpha=0.05$ ) between baseline and CCI $(p=0.05$; Cohen's $d$ values $=2.87)$, but not between baseline and saline $(p=0.2)$ or between baseline and muscimol $(p>0.99)$. Right face (contralateral) mechanical withdrawal thresholds did not change across time points

\footnotetext{
$\leftarrow$

images of coronal sections through CeA demonstrate that CeA-LPB neurons can express somatostatin, CRH, or dynorphin mRNA. Large panels, Representative high-magnification images of merged signal from DAPI (pan-cellular stain; blue), somatostatin (green), FG (white), and (RH (red) in $\mathbf{C}$ or dynorphin (red) in $\boldsymbol{D}$. White arrows indicate neurons that are $\mathrm{FG}^{+}$and that colabel with one or both of the assayed mRNAs. Small panels, Individual signals seen in the respective large panel. White arrows confirm neurochemical phenotype. $\boldsymbol{E}$, $\boldsymbol{F}$, Mean and $95 \% \mathrm{Cl}$ for the fraction of $\mathrm{FG}^{+}$neurons that colabeled for somatostatin, dynorphin, or both $(\boldsymbol{E})$, and somatostatin, $\mathrm{CRH}$, or both $(\boldsymbol{F})$ as well as the total fraction of identified and unidentified $\mathrm{FG}^{+}$neurons per assay. Scale bars, $250 \mu \mathrm{m}$.
}

(Friedman's $F=6.830 ; p=0.07$; Fig. $7 C$ ). Median mechanical withdrawal thresholds were $34.8 \mathrm{~g}$ at baseline $(95 \% \mathrm{CI}=32.5-$ $34.8 \mathrm{~g}), 24.2 \mathrm{~g}$ after CCI (95\% CI $=17.0-32.5 \mathrm{~g}), 22.7 \mathrm{~g}$ after saline $(95 \% \mathrm{CI}=10.7-37.0 \mathrm{~g})$, and $32.5 \mathrm{~g}$ after muscimol $(95 \%$ $\mathrm{CI}=27.2-37.0 \mathrm{~g})$.

Withdrawal thresholds to mechanical stimuli applied to both hindpaws were affected by muscimol but not saline (Fig. $7 D, E$ ). Left hindpaw mechanical withdrawal thresholds changed across time points (Friedman's $F=12.60 ; p=0.0006$; Fig. $7 D$ ). Median mechanical withdrawal thresholds changed from $66.6 \mathrm{~g}$ at baseline $(95 \% \mathrm{CI}=62.9-70.3 \mathrm{~g})$ to $22.9 \mathrm{~g}$ after CCI $(95 \% \mathrm{CI}=21.4-51.4 \mathrm{~g}), 31.4 \mathrm{~g}$ after saline infusion $(95 \%$ $\mathrm{CI}=11.9-39.5 \mathrm{~g})$, and $62.5 \mathrm{~g}$ after muscimol application $(95 \%$ $\mathrm{CI}=51.8-70.3 \mathrm{~g}$ ). Individual comparisons (using Dunn's correction for multiple comparisons) revealed a difference between baseline and CCI $(p=0.02$; Cohen's $d$ values $=3.51)$, baseline and saline ( $p=0.0099$; Cohen's $d$ values $=4.15)$, but not between baseline and muscimol ( $p>0.99$; Cohen's $d$ values $=0.80$ ).

We observed similar results on the contralateral hindpaw (Fig. 7E). Right hindpaw mechanical withdrawal thresholds changed across time points (Friedman's $F=13.04 ; p=0.0003$; Fig. 7E). Median mechanical withdrawal thresholds changed from $66.6 \mathrm{~g}$ at baseline (95\% CI $=66.6-70.3 \mathrm{~g}$ ) to $22.2 \mathrm{~g}$ after CCI (95\% CI = 19.1-51.4 g), $37.7 \mathrm{~g}$ after saline application $(95 \%$ $\mathrm{CI}=34.9-55.1 \mathrm{~g}$ ), and $66.6 \mathrm{~g}$ after muscimol infusion $(95 \%$ $\mathrm{CI}=59.2-75.0 \mathrm{~g}$ ). Individual comparisons (after Dunn's correction) revealed a difference between baseline and CCI ( $p=0.007$; Cohen's $d$ values $=3.19$ ), but not between baseline and saline $(p=0.06)$ or baseline and muscimol $(p>0.99)$.

RGS scores, reflecting ongoing ("spontaneous") pain, changed across time points (Friedman's $F=14.04 ; p<0.0001$; Fig. $7 F$ ), from a median of $0.12 \mathrm{AU}$ at baseline $(95 \% \mathrm{CI}=0.06-0.34 \mathrm{AU})$ to $0.81 \mathrm{AU}$ after CCI ( $95 \% \mathrm{CI}=0.25-0.96 \mathrm{AU})$. After CCI, infusion of saline caused a small decrease to $0.57 \mathrm{AU}(95 \% \mathrm{CI}=0.07$ $0.71 \mathrm{AU}$ ), whereas infusion of muscimol caused a dramatic decrease to $0.21 \mathrm{AU}(95 \% \mathrm{CI}=0.10-0.57 \mathrm{AU})$. Individual comparisons (using Dunn's corrections) revealed a difference between baseline and CCI $(p=0.0007$; Cohen's $d$ values = $-2.35)$, but not between baseline and saline $(p=0.08)$, or baseline and muscimol $(p=0.4)$.

These findings indicate that pharmacological inhibition of $\mathrm{PB}$ attenuates both reflexive and ongoing signs of chronic pain.

\section{Pharmacological disinhibition of $P B$}

We tested also the corollary prediction that disinhibition of $\mathrm{PB}$ will amplify behavioral metrics of pain. We did this by infusing gabazine, a $\mathrm{GABA}_{\mathrm{A}}$ receptor antagonist, into $\mathrm{LPB}$ of naive rats. Consistent with previous reports (Han et al., 2015; Barik et al., 2018; Chiang et al., 2019), this procedure appeared to be aversive to most animals, often resulting in either freezing or escape behaviors. This made it difficult for us to quantify pain-related behaviors in most of these animals.

In a subset of these animals, we were able to evaluate hindpaw mechanical withdrawal thresholds (Fig. $7 H, I$ ). In the left hindpaw, the thresholds of gabazine-treated animals $(n=7)$ were reduced to 0.65 of baseline values $(95 \% \mathrm{CI}=0.32-0.94$; Fig. $7 H$ ), whereas ratios for saline controls $(n=6)$ remained unchanged at 1.00 (95\% CI $=0.95-1.06$; Mann-Whitney $U=0 ; p=0.0012$; Cohen's $d$ values $=2.03$ ). In the right hindpaw, ipsilateral to the gabazine injections, mechanical thresholds of gabazine-treated animals were reduced to a median of $0.63(95 \% \mathrm{CI}=0.24-0.95$; Fig. $7 I)$, whereas saline controls had a median ratio of $1.05(95 \%$ 
A

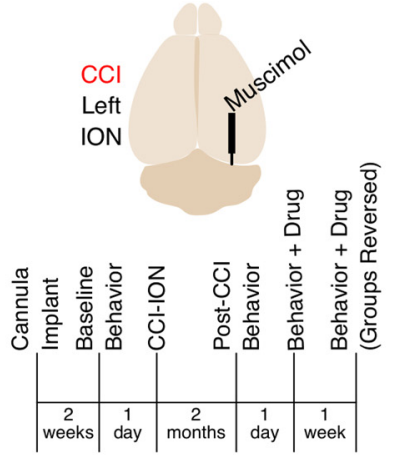

D

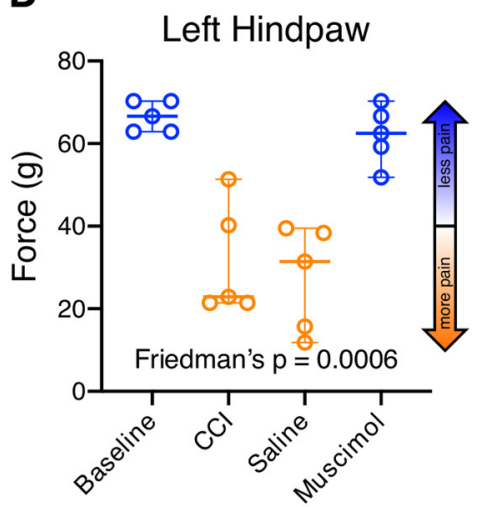

G

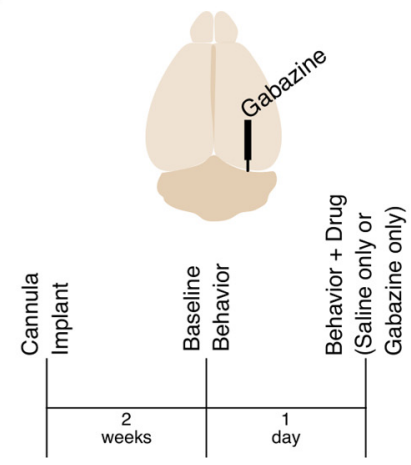

B

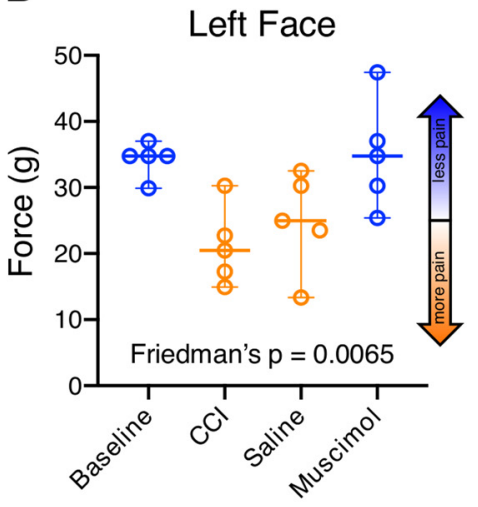

E

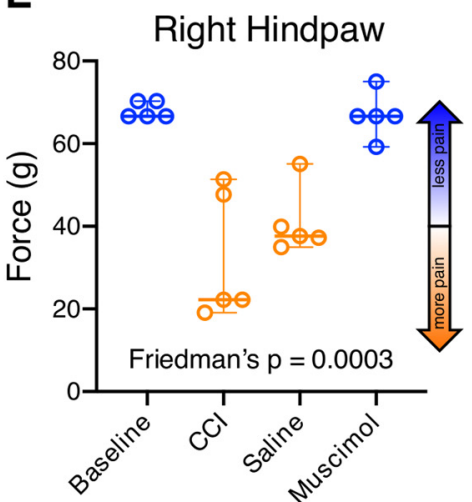

H

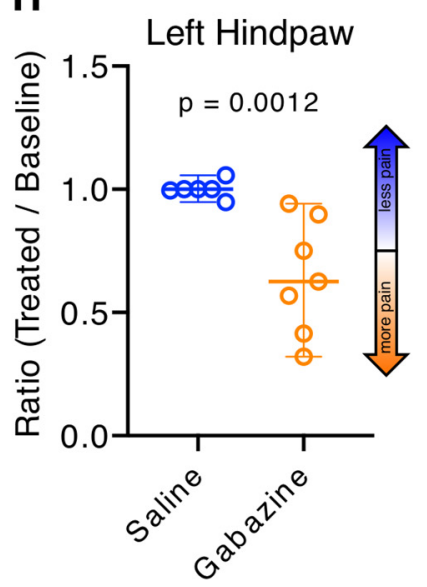

C

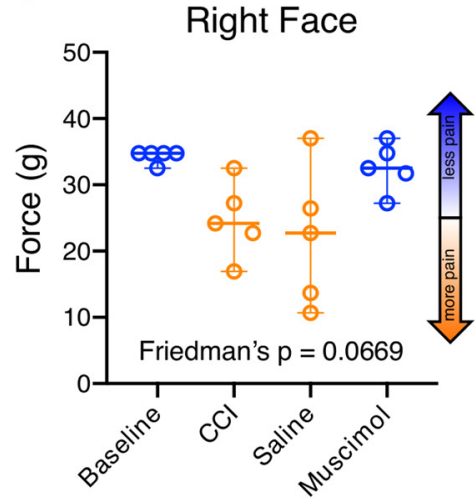

$\mathbf{F}$

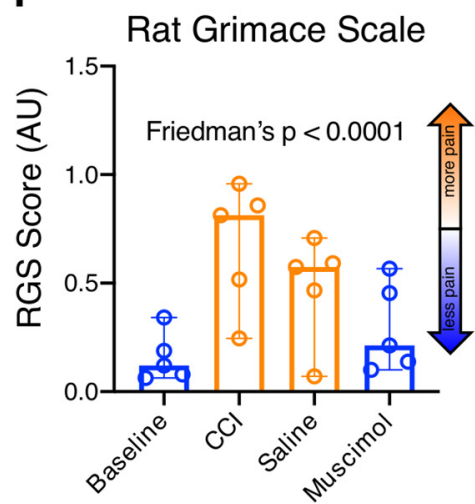

I

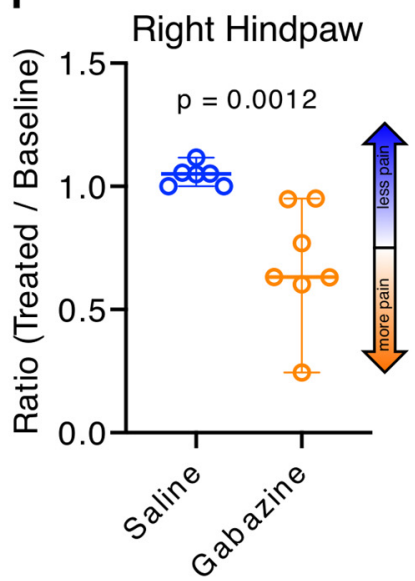

Figure 7. Pharmacologically inhibiting PB suppresses CCI-ION pain. In naive animals, disinhibiting PB amplifies acute reflexive pain. Data are medians and $95 \%$ Cls. $\boldsymbol{A}$, Timeline of injury, cannula implantation, behavioral testing, and PB inhibition experimental design. $\boldsymbol{B}-\boldsymbol{F}, \mathrm{A}$ single infusion of muscimol into the right $\mathrm{LPB}$ ameliorated the effect of $\mathbf{C C I}$-ION on mechanical withdrawal thresholds in the left face $(\boldsymbol{B})$ and both hindpaws $(\boldsymbol{D}, \boldsymbol{E})$, but not in the right face $(\boldsymbol{C})$. Spontaneous pain RGS scores were also reduced by muscimol but unaffected by saline $(\boldsymbol{F})$. $\boldsymbol{G}$, Timeline of cannula implantation, behavioral testing, and PB disinhibition experimental design. $\boldsymbol{H}, \boldsymbol{I}, \mathbf{A}$ single infusion of gabazine, but not saline, reduced hindpaw mechanical withdrawal thresholds both ipsilateral $(\boldsymbol{I})$ and contralateral $(\boldsymbol{H})$ to LPB cannulation.

$\mathrm{CI}=1.00-1.12$; Mann-Whitney $U=0 ; p=0.0012$; Cohen's $d$ values $=2.00)$. These findings suggest that unilateral disinhibition of $\mathrm{PB}$ results in reduced mechanical withdrawal thresholds to stimuli applied bilaterally to the hindpaws.

\section{Optogenetics}

Our findings that inhibiting PB attenuates chronic pain, and that CeA provides the major inhibitory input to $\mathrm{PB}$, suggest that the inhibitory CeA-PB pathway is causally involved in regulating the experience of perception of pain and aversion. To test this hypothesis, we optogenetically excited CeA axon terminals in LPB while monitoring behavioral metrics of pain. We injected hChR2 viral constructs (see Materials and Methods) into the right CeA of WT or transgenic CRH-Cre rats, and implanted fiberoptic cannulae over the right LPB. Although the fluorescent reporter protein expressed by the viral construct can be observed in LPB at 6 weeks, expression appears to peak and stabilize at $\sim 9$ weeks after injection (similar to previous reports, Pomrenze et al., 2015). We used animals of both sexes but did not have sufficient sample sizes to test for sex differences. To avoid potential confounds related to long-duration optogenetic stimulation (Tyssowski and Gray, 2019), we delivered short pulses of $470 \mathrm{~nm}$ 
A
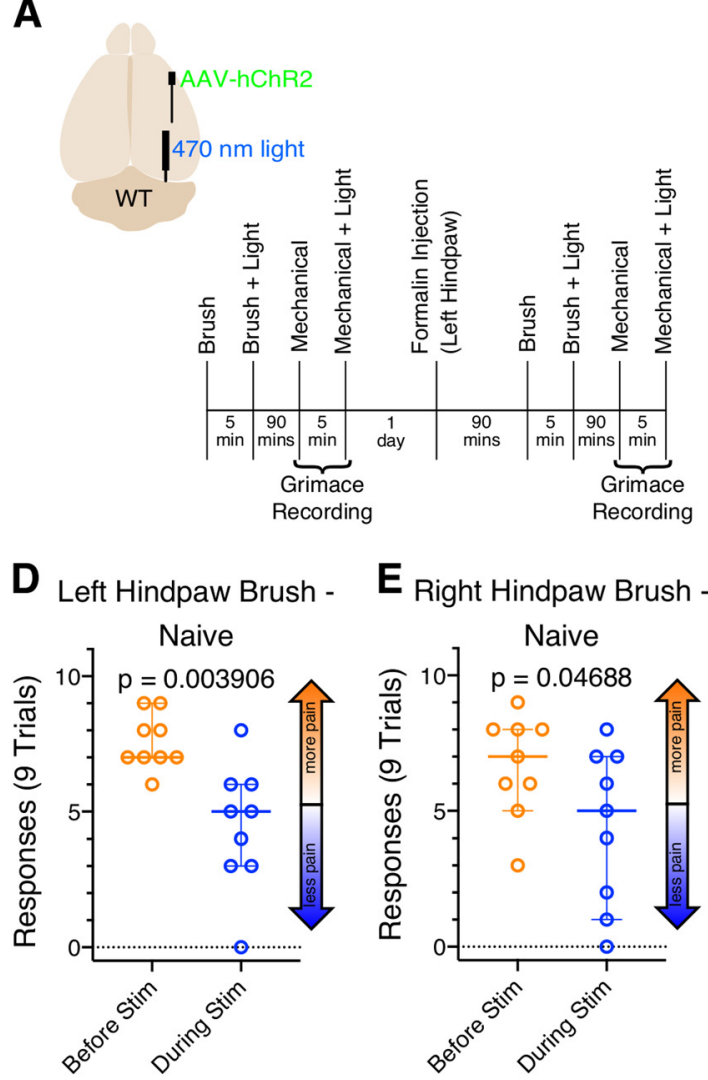

H Left Hindpaw Brush -
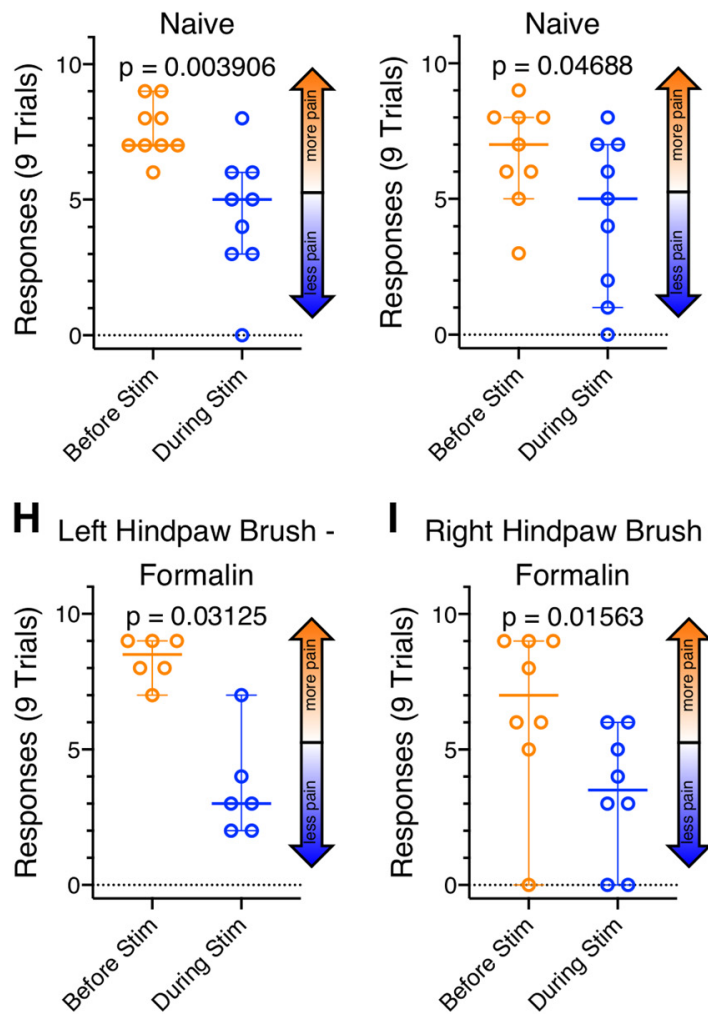

B

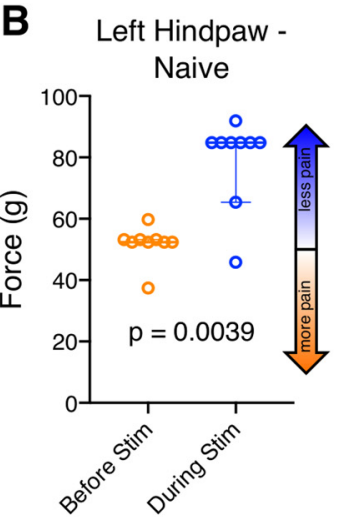

E Right Hindpaw Brush -

I Right Hindpaw Brush -

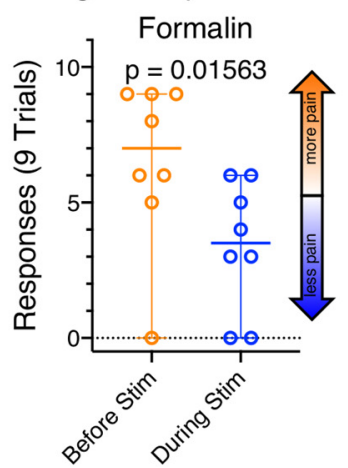

$\mathbf{F}$

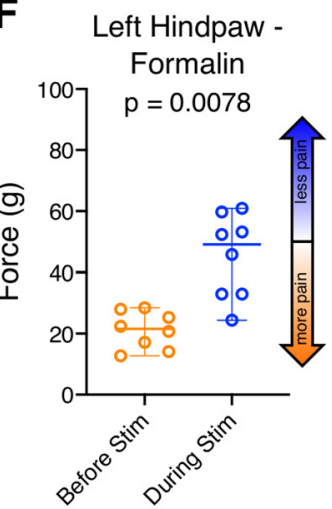

J Rat Grimace Scale -

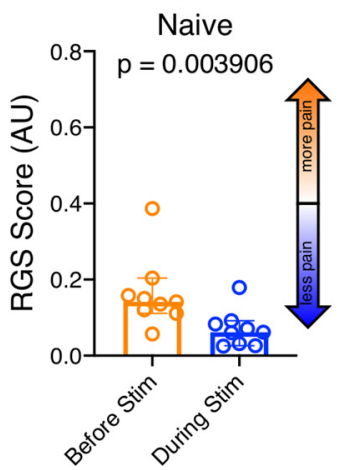

C Right Hindpaw Naive

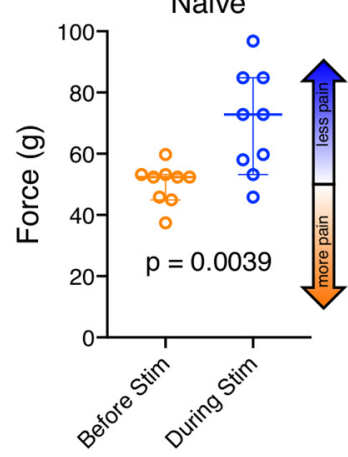

G Right Hindpaw Formalin

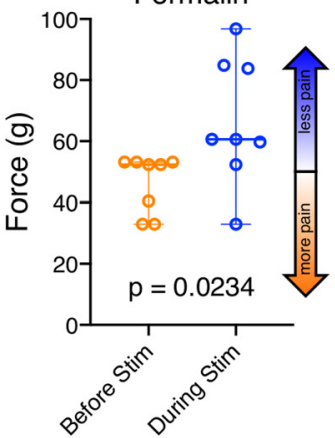

K Rat Grimace Scale Formalin

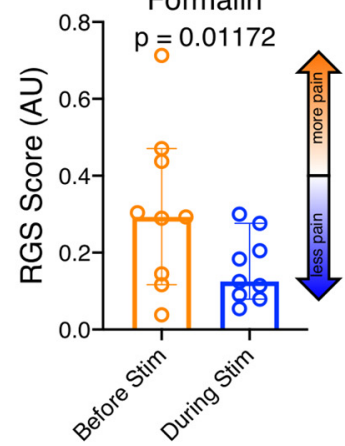

Figure 8. Optogenetically activating CeA-LPB terminals suppresses pain in the naive animal and during acute formalin pain. $A$, Locations of viral construct injection, fiberoptic implantation, and experimental design. $\boldsymbol{B}-\boldsymbol{K}$, Data are medians and $95 \%$ Cls. Activating right side CeA-LPB terminals using short pulses of light increased mechanical withdrawal thresholds in both hindpaws $(\boldsymbol{B}, \boldsymbol{C})$ and decreased both mechanical allodynia responses $(\boldsymbol{D}, \boldsymbol{E})$ as well as spontaneous pain RGS scores $(\boldsymbol{V})$ in the naive animal. During acute formalin pain, optogenetically activating CeA-LPB terminals also increased hindpaw mechanical withdrawal thresholds $(\boldsymbol{F}, \boldsymbol{G})$ and decreased both mechanical allodynia responses $(\boldsymbol{H}, \boldsymbol{I})$ as well as RGS scores $(\boldsymbol{K})$.

light (a single train of 5 light pulses, $4 \mathrm{~ms}$ duration/pulse, $10 \mathrm{~Hz}$ ) only during application of mechanical or dynamic stimuli.

\section{Mechanical withdrawal}

We first tested the effects of activating CeA-LPB terminals in PB on mechanical allodynia in WT animals. Figure $8 B, C$ depicts the hindpaw mechanical withdrawal thresholds for individual animals $(n=9)$ as well as the median and $95 \%$ CIs for each time point. Left hindpaw thresholds increased from a median of $52.3 \mathrm{~g}$ at baseline (95\% CI $=52.3-53.2 \mathrm{~g}$ ) to $84.8 \mathrm{~g}$ during optical stimulation (95\% CI $=65.4-84.8 g$; Wilcoxon $p=0.004$; Cohen's $d$ values $=$ 2.49; Fig. $8 B$ ). Similarly, right hindpaw thresholds increased from a median of $52.3 \mathrm{~g}$ at baseline $(95 \% \mathrm{CI}=44.9-53.2 \mathrm{~g})$ to $72.8 \mathrm{~g}$ during optical stimulation $(95 \% \mathrm{CI}=53.2-84.8 g$; Wilcoxon $p=0.004$; Cohen's $d$ values $=1.54$; Fig. $8 C$ ).

We repeated this experiment in CRH-Cre animals, in which hChR2 is expressed only in the subset of GABAergic CeA neurons expressing $\mathrm{CRH}$. As in WT animals, left hindpaw mechanical withdrawal thresholds increased from a median of $53.2 \mathrm{~g}$ at baseline $(95 \% \mathrm{CI}=37.5-72.8 \mathrm{~g})$ to $84.8 \mathrm{~g}$ during optical stimulation (95\% CI $=52.3-96.8 g$; Wilcoxon $p=0.016$; Cohen's $d$ values $=1.81$; Fig. $9 B$ ). Similarly, right hindpaw thresholds increased from a median of $52.3 \mathrm{~g}$ at baseline $(95 \%$ $\mathrm{CI}=37.5-60.6 \mathrm{~g})$ to $84.8 \mathrm{~g}$ during optical stimulation $(95 \%$ $\mathrm{CI}=44.9-96.8 g$; Wilcoxon $p=0.016$; Cohen's $d$ values $=1.68$; Fig. 9C). 
A

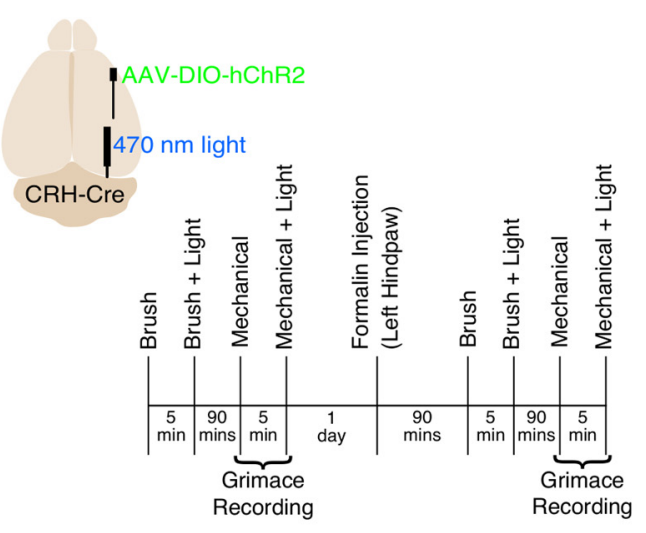

B

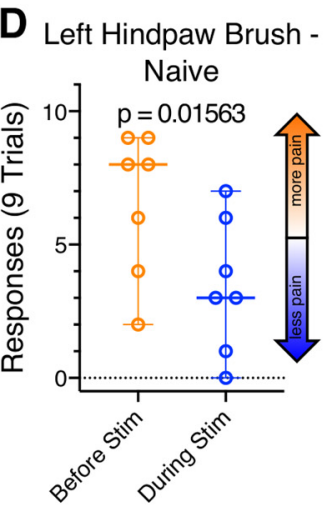

H Left Hindpaw Brush -

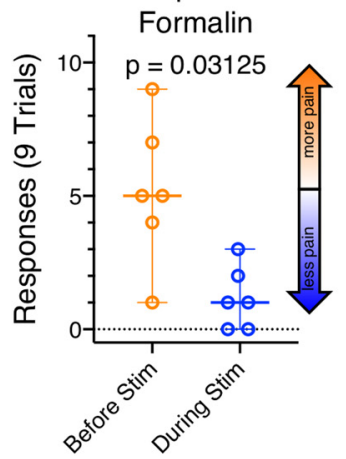

$\mathbf{E}_{\text {Right Hindpaw Brush - }}$

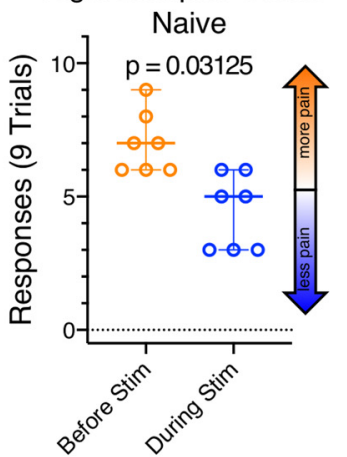

I Right Hindpaw Brush -

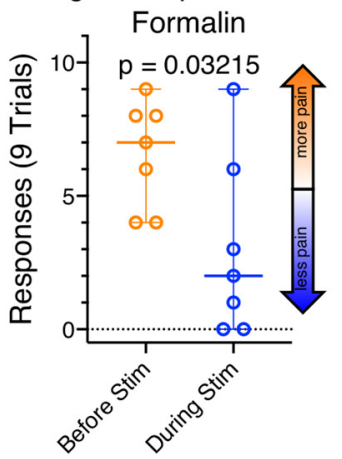

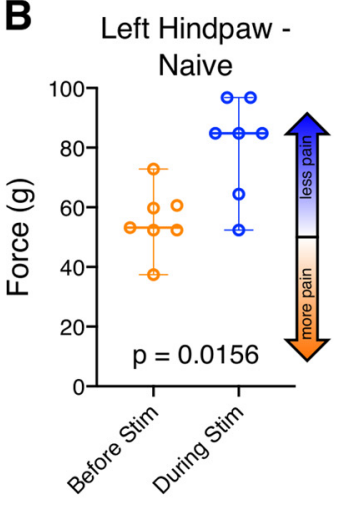

$F$

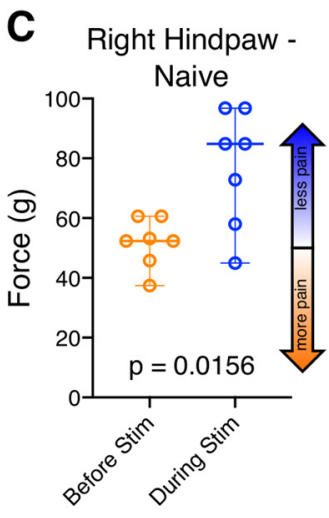

G Right Hindpaw -

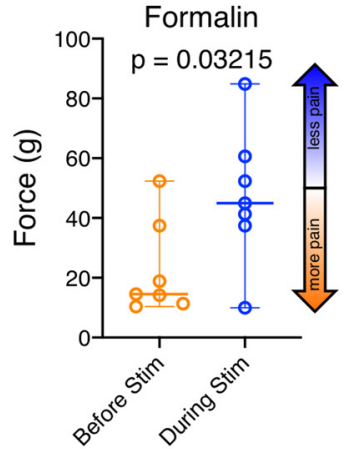

J Rat Grimace Scale -

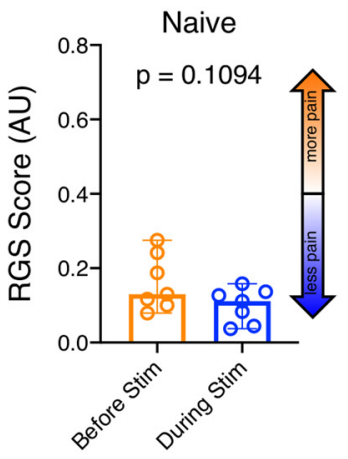
Formalin

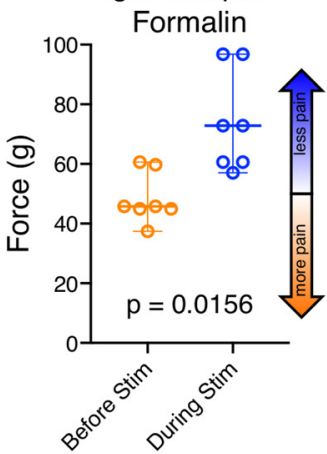

K Rat Grimace Scale -

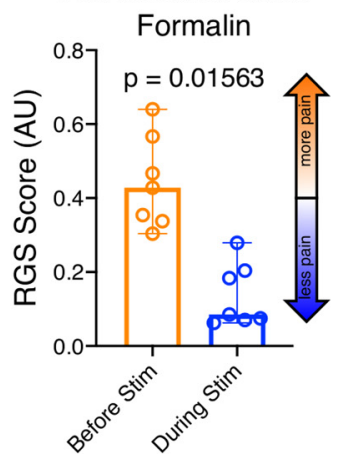

Figure 9. Optogenetically activating a subset of GABAergic CeA-LPB axon terminals in CRH-Cre rats suppresses pain in naive animals, as well as during acute formalin pain. $A$, Locations of viral construct injection, fiberoptic implantation, and experimental design. $\boldsymbol{B}-\boldsymbol{K}$, Data are medians and $95 \%$ Cls. Selectively activating CRH CeA terminals in LPB increased mechanical withdrawal thresholds in both hindpaws $(\boldsymbol{B}, \boldsymbol{C})$ and decreased mechanical allodynia responses $(\boldsymbol{D}, \boldsymbol{E})$, but did not affect spontaneous pain RGS scores $(\boldsymbol{H})$ in the naive animal. During acute formalin pain, optogenetically activating this subset of CeA-LPB axon terminals also increased hindpaw mechanical withdrawal thresholds $(\boldsymbol{F}, \boldsymbol{G})$ and decreased both mechanical allodynia responses $(\boldsymbol{H}, \boldsymbol{I})$ as well as RGS scores $(\boldsymbol{K})$.

\section{Dynamic mechanical allodynia}

Activating CeA-LPB terminals in PB also reduced responses to the hindpaw brush test (see Materials and Methods). Figure $8 D$, $E$ depicts the number of times each animal responded to the brush stimuli. In WT animals $(n=9)$, left hindpaw brush responses decreased from a median of 7 of 9 stimuli at baseline (95\% CI $=7-9$ responses) to 5 responses during optical stimulation (95\% CI $=3-6$; Wilcoxon $p=0.004$; Cohen's $d$ values $=1.75$; Fig. $8 D$ ). Similarly, right hindpaw brush response decreased from a median of 7 responses at baseline $(95 \% \mathrm{CI}=5-8$ responses) to 5 responses during optical stimulation (95\% $\mathrm{CI}=1-7$ responses; Wilcoxon $p=0.047$; Cohen's $d$ values $=0.92$; Fig. $8 E$ ).

In CRH-Cre animals $(n=7)$, left hindpaw brush response decreased from a median of 8 responses of 9 stimulus applications at baseline (95\% $\mathrm{CI}=2-9$ responses) to 3 responses during optical stimulation (95\% $\mathrm{CI}=0-7$ responses; Wilcoxon $p=0.016$; Cohen's $d$ values $=1.21$; Fig. $9 D$ ). Similarly, right hindpaw brush response decreased from a median of 7 responses at baseline (95\% CI $=6-9$ responses) to 5 responses during optical stimulation (95\% $\mathrm{CI}=3-6$ responses; Wilcoxon $p=0.03$; Cohen's $d$ values $=2.01$; Fig. $9 E$ ) .

\section{Formalin-induced pain}

To test the effects of activating the CeA-PB pathway on ongoing pain, we injected, $1 \mathrm{~d}$ after baseline testing, $5 \%$ formalin $(50 \mu \mathrm{l})$ into the dorsal surface of the left hindpaw (contralateral to CeA virus injection and LPB cannula implantation). Left hindpaw mechanical withdrawal thresholds increased from a median of $21.6 \mathrm{~g}$ after formalin exposure $(95 \% \mathrm{CI}=12.7-28.5 \mathrm{~g})$ to $49.1 \mathrm{~g}$ 
during optical stimulation (95\% CI $=24.4-60.9 g$; Wilcoxon $p=0.008$; Cohen's $d$ values $=2.29$; Fig. $8 F$ ). Similarly, right hindpaw thresholds increased from a median of $52.3 \mathrm{~g}$ after formalin exposure $(95 \% \mathrm{CI}=33.0-53.2 \mathrm{~g})$ to $60.6 \mathrm{~g}$ during optical stimulation (95\% CI $=33.0-96.8 g$; Wilcoxon $p=0.02$; Cohen's $d$ values $=1.25$; Fig. $8 G)$.

Similar effects of optogenetic activation occurred in CRH-Cre rats $(n=7$; Fig. 9F, $G$ ). Left hindpaw withdrawal thresholds increased from a median of $14.5 \mathrm{~g}$ after formalin exposure (95\% $\mathrm{CI}=10.4-52.3 \mathrm{~g})$ to $44.9 \mathrm{~g}$ during optical stimulation $(95 \%$ $\mathrm{CI}=10.0-84.8 g$; Wilcoxon $p=0.03$; Cohen's $d$ values $=1.25$; Fig. $9 F)$. Similarly, right hindpaw thresholds increased from a median of $45.8 \mathrm{~g}$ after formalin exposure $(95 \% \mathrm{CI}=37.5-60.6 \mathrm{~g})$ to $72.8 \mathrm{~g}$ during optical stimulation $(95 \% \mathrm{CI}=57.0-96.8 \mathrm{~g}$; Wilcoxon $p=0.016$; Cohen's $d$ values $=1.91$; Fig. $9 G)$.

Optical stimulation of the CeA-PB pathway also attenuated dynamic mechanical allodynia evoked by the formalin injection. After formalin injection in the left hindpaw of WT animals ( $n=6$ animals), brush response to the same paw decreased from a median of 8.5 responses of 9 stimuli $(95 \% \quad \mathrm{CI}=7-9$ responses) to 3 responses during optical stimulation $(95 \%$ $\mathrm{CI}=2-7$; Wilcoxon $p=0.0312$; Cohen's $d$ values $=3.35$; Fig. $8 H$ ). Similarly, right hindpaw brush response ( $n=8$ animals) decreased from a median of 7 responses after formalin exposure $(95 \% \mathrm{CI}=0-9$ responses) to 3.5 responses during optical stimulation (95\% CI $=0-6$; Wilcoxon $p=0.0156$; Cohen's $d$ values = 1.14; Fig. $8 I$ ).

We obtained similar results in CRH-Cre animals ( $n=6$; Fig. $9 H, I)$. Left hindpaw brush responses decreased from a median of 5 of 9 stimuli after formalin exposure ( $95 \% \mathrm{CI}=1-9$ responses) to 1 response during optical stimulation $(95 \% \quad \mathrm{CI}=0-3$ responses; Wilcoxon $p=0.03$; Cohen's $d$ values $=1.91$; Fig. $9 H$ ). Similarly, right hindpaw brush response ( $n=7$ animals) decreased from a median of 7 of 9 responses after formalin exposure (95\% CI $=4-9$ responses) to 2 responses during optical stimulation ( $95 \% \mathrm{CI}=0-9$ responses; Wilcoxon $p=0.03$; Cohen's $d$ values $=1.29$; Fig. $9 I)$.

\section{Grimace}

Stimulation of the amygdalo-parabrachial also attenuated RGSs in rats with formalin-induced pain (Fig. 8J, K). In naive animals $(n=9)$, activating CeA-LPB reduced median RGS scores from $0.14 \mathrm{AU}(95 \% \mathrm{CI}=0.11-0.20 \mathrm{AU}$ ) at baseline to 0.06 AU (95\% $\mathrm{CI}=0.03-0.09 \mathrm{AU})$ during light stimulation (Wilcoxon $p=0.004$; Cohen's $d$ values $=1.26$; Fig. $8 J$ ). After formalin injection, activation of CeA-PB reduced median RGS scores from $0.29 \mathrm{AU}(95 \% \mathrm{CI}=0.12-0.47 \mathrm{AU})$ during acute formalin pain to $0.12 \mathrm{AU}(95 \% \mathrm{CI}=0.08-0.28 \mathrm{AU})$ during optical stimulation (Wilcoxon $p=0.01$; Cohen's $d$ values $=0.96$; Fig. $8 K)$.

We replicated these results in CRH-Cre animals ( $n=7$; Fig. $9 J, K)$. In naive animals, activating CeA-LPB reduced median RGS scores only nominally, from 0.13 AU (95\% CI $=0.08$ $0.27 \mathrm{AU})$ at baseline to $0.11 \mathrm{AU}(95 \% \mathrm{CI}=0.04-0.16 \mathrm{AU})$ during light stimulation (Wilcoxon $p=0.11$; Fig. $9 J$ ). However, after formalin injection, activation of CeA-LPB reduced median RGS scores from $0.43 \mathrm{AU}(95 \% \mathrm{CI}=0.30-0.64 \mathrm{AU})$ during acute formalin pain to $0.08 \mathrm{AU}(95 \% \mathrm{CI}=0.06-0.28 \mathrm{AU})$ during optical stimulation (Wilcoxon $p=0.016$; Cohen's $d$ values $=2.86$; Fig. 9K).

Together, these findings suggest that optogenetic activation of the CeA-PB pathway inhibits LPB neurons, resulting in attenuation of both acute and ongoing pain-related behaviors.

\section{Discussion}

\section{Amplified activity of PB neurons}

As detailed in the Introduction, the PB complex, and, in particular, its lateral division (LPB), is a critical nexus for processing nociception and for the perception of both sensory and affective components of pain. Growing evidence supports the notion that these pain-related functions are part of a larger role of $\mathrm{PB}$ in mediating aversion (see Introduction).

Here, we report that a mouse model of chronic, neuropathic pain, CCI-ION, results in lasting amplification of activity in LPB neurons. This amplification was expressed as increased spontaneous activity and larger magnitude responses to noxious stimuli. Particularly striking was the increase in ADs, responses that far outlast stimuli. ADs have been described in other brain regions after chronic pain (Woolf and King, 1987; Herrero et al., 2000). Their durations, and the proportion of neurons that express them, are dramatically increased in chronic pain (Palecek et al., 1992; Laird and Bennett, 1993). ADs are also key in "windup," the progressively increased excitability of spinal neurons that occurs in central sensitization to pain (Morisset and Nagy, 2000). ADs may increase pain perception by enhancing the transfer of nociceptive responses to downstream structures (Morisset and Nagy, 1998). There is compelling evidence that $\mathrm{ADs}$ are causally related to the presence of chronic pain (Laird and Bennett, 1993; Asada et al., 1996). For example, we have demonstrated that the incidence and duration of ADs in spinal neurons increase significantly in animals with chronic, neuropathic pain. Importantly, suppressing ADs significantly lessens hyperalgesia in experimental animals (Okubo et al., 2013).

The current finding in mice is similar to that in our previous study in rats, in which we reported a dramatic increase in ADs of PB neurons after CCI-ION (Uddin et al., 2018). However, contrary to our current findings, in the rat, CCI-ION was not associated with changes in spontaneous firing rates of $\mathrm{PB}$ neurons, or in their responses during stimulus application. We do not yet know whether these species differences reflect methodological differences, or more fundamental biological distinctions.

\section{Disinhibition of $\mathrm{PB}$ neurons}

The amplification of $\mathrm{PB}$ neuronal activity was not related to changes in passive membrane properties, as neither the input resistance nor the resting membrane potential of $\mathrm{PB}$ neurons was affected by CCI-ION. What did change dramatically was the inhibitory input to these neurons. The frequency of mIPSCs was reduced by half, whereas the amplitude of mIPSCs remained unchanged, suggesting a reduction in presynaptic inhibitory inputs to $\mathrm{PB}$ neurons. That reduced inhibition in $\mathrm{PB}$ is related to chronic pain is consistent with findings in other CNS regions, where similar disinhibitory mechanisms are causally related to chronic pain (for review, see Prescott, 2015; Todd, 2015). Reduced inhibition may directly result in the increased spontaneous and evoked responses in mouse $\mathrm{PB}$ neurons in chronic pain, as reported here.

It remains to be determined whether disinhibition gives rise to the pronounced ADs in $\mathrm{PB}$ neurons of rodents with chronic pain. In other neuronal populations, ADs appear to be mediated by interactions between synaptic inhibition, NMDARs, and potassium currents (Traub et al., 1995; Sotgiu and Biella, 2002; Drew et al., 2004). Whether similar biophysics governs that pathophysiology of ADs in $\mathrm{PB}$ during chronic pain remains to be determined. 


\section{Source of PB inhibition}

We find that a relatively modest proportion of neurons (12\% in rats) in $\mathrm{PB}$ are GABAergic. This is consistent with previous reports of a population of inhibitory neurons in the rodent $\mathrm{PB}$ (Chen et al., 2017; Chiang and Ross, 2017; Geerling et al., 2017; Chiang et al., 2019). Here, we focused on the role of extrinsic GABAergic inputs to $\mathrm{PB}$.

\section{The CeA-PB pathway}

We identified several extrinsic sources of GABAergic inputs to $\mathrm{PB}$, including the zona incerta, hypothalamus, and, most prominent, the CeA. Our results show that this inhibitory CeA-PB amygdalo- $\mathrm{PB}$ pathway plays a key role in regulating pain-related activity involving $\mathrm{PB}$.

It is well established that $\mathrm{CeA}$ is a central node for pain processing, integrating nociception, contexts, affect, and memory to guide behaviors related to acute and chronic pain (Davis, 1994; Zald, 2003; Veinante et al., 2013; Neugebauer, 2015; Woodhams et al., 2017; Wilson et al., 2019).

We describe a dense, GABAergic pathway, originating from CeA and innervating $\mathrm{PB}$. This pathway exists in both rats and mice and provides potent inhibition to $\mathrm{PB}$ neurons. We show that, in both rodent species, this amygdalo-parabrachial inhibitory pathway regulates responses of $\mathrm{PB}$ neurons to noxious stimuli and behaviors evoked by these stimuli. We also demonstrate that activating this inhibitory pathway ameliorates signs of chronic pain, and that suppressing this pathway in naive animals promotes pain behaviors. These findings support a causal role for the amygdalo-parabrachial pathway in both acute and chronic pain.

Our findings are consistent with prior anatomic reports that made reference to a direct projection from CeA to PB (Jia et al., 1994; Sun et al., 1994; Chieng et al., 2006; Pomrenze et al., 2015). Jia et al. (2005) provided a more extensive description of afferents originating from $\mathrm{CeA}$ and terminating in the LPB, where they form symmetrical, presumably inhibitory, synapses. More recently, Torruella-Suárez et al., (2019) reported that inhibitory, neurotensin-expressing CeA neurons densely innervate and inhibit $\mathrm{PB}$, and that stimulation of these projections promotes consumption of ethanol and palatable fluids.

The GABAergic projections from CeA to $\mathrm{PB}$ complement the more extensively described reciprocal projections from $\mathrm{PB}$ to CeA (Saper and Loewy, 1980; Bernard et al., 1993; Sarhan et al., 2005), which are involved in mediating pain-related behaviors (Han et al., 2015). This $\mathrm{PB} \rightarrow \mathrm{CeA}$ pathway is a glutamatergic, excitatory one (Neugebauer, 2015), balanced by the inhibitory reciprocal pathway from $\mathrm{CeA}$ to $\mathrm{PB}$ described here. At least some $\mathrm{PB}$ neurons that project to $\mathrm{CeA}$ appear to receive inhibitory inputs from $\mathrm{CeA}$, consistent with direct connections between individual $\mathrm{CeA} \rightarrow \mathrm{PB}$ and $\mathrm{PB} \rightarrow \mathrm{CeA}$ neurons (Jia et al., 2005). $\mathrm{PB}$ projections target both somatostatin and $\mathrm{CRH}$ neurons in specific subdivisions of $\mathrm{CeA}$; in a model of chronic pain, the strengths of these projections may increase or decrease, depending on the neuronal population targeted in CeA ( $\mathrm{Li}$ and Sheets, 2020). How these reciprocal pathways influence each other remains to be determined. We discuss several possibilities below.

Consistent with previous reports that essentially all CeA neurons are inhibitory (Ehrlich et al., 2009; Janak and Tye, 2015), we find that $\mathrm{CeA}$ neurons that project to $\mathrm{PB}$ are GABAergic. CeA neurons are functionally and neurochemically heterogeneous (Janak and Tye, 2015; Wilson et al., 2019). We find that the GABAergic CeA neurons involved in the CeA-PB pathway are also heterogeneous and include neurons that express dynorphin
(42\% of these projections neurons), somatostatin (26\%), and CRH (9\%), with some expressing more than one of these substances. We did not probe for other neurochemical phenotypes expressed in CeA, such as $\mathrm{PKC} \delta$ (Wilson et al., 2019) or neurotensin (Torruella-Suárez et al., 2019). How these diverse populations of CeA-PB neurons regulate nociception and pain remains to be fully elucidated.

At least one of these populations, the somatostatin-expressing neurons, has been directly implicated in these functions, as they are inhibited by nerve injury, and activating them attenuates mechanical allodynia (Wilson et al., 2019). These somatostatin CeA neurons are involved also in responses to threat-related sensory cues (Yu et al., 2016; Fadok et al., 2017). Wilson et al. (2019) demonstrated that two populations of CeA neurons are involved in opposing functions in pain regulation. $\mathrm{PKC} \delta$-expressing neurons are involved in functions that promote pain (pronociceptive), whereas somatostatin-CeA neurons are involved in opposing, antinociceptive functions. Our present results suggest that the antinociceptive function of these somatostatin-CeA neurons is accomplished by their direct, inhibitory action on $\mathrm{PB}$ neurons.

The particularly large population of CeA-PB neurons that express dynorphin is also of significance because the dynorphin receptor, the $\kappa$-opioid receptor, is highly expressed in $\mathrm{PB}$ (Unterwald et al., 1991; Yasuda et al., 1993; Mansour et al., 1994). How dynorphin regulates PB neuronal activity is still unknown. By analogy to other CNS structures, where these functions are better understood in the context of pain (Bie and Pan, 2003; Wang et al., 2018; Navratilova et al., 2019), we speculate that dynorphin, acting on $\kappa$-opioid receptors, may directly inhibit PB neurons thorough postsynaptic receptors, as well as regulate GABA release presynaptically. We are currently studying these mechanisms.

Together, our findings demonstrate a hitherto unknown pathway of pain regulation, involving two key structures associated primarily with the affective aspects of chronic pain: the $\mathrm{PB}$ complex and the amygdala. Because the affective component of chronic pain is the component most directly associated with patients' suffering, and is the component most resistant to treatment (Han et al., 2015; Neugebauer, 2015; Price et al., 2018; Corder et al., 2019), the identification of a novel pathway mediating this function may be directly relevant to planning novel therapies for this devastating condition.

\section{References}

Akintola T, Raver C, Studlack P, Uddin O, Masri R, Keller A (2017) The grimace scale reliably assesses chronic pain in a rodent model of trigeminal neuropathic pain. Neurobiol Pain 2:13-17.

Akintola T, Tricou C, Raver C, Castro A, Colloca L, Keller A (2019) In search of a rodent model of placebo analgesia in chronic orofacial neuropathic pain. Neurobiol Pain 6:100033.

Asada H, Yamaguchi Y, Tsunoda S, Fukuda Y (1996) Relation of abnormal burst activity of spinal neurons to the recurrence of autotomy in rats. Neurosci Lett 213:99-102.

Auvray M, Myin E, Spence C (2010) The sensory-discriminative and affective-motivational aspects of pain. Neurosci Biobehav Rev 34:214-223.

Barik A, Thompson JH, Seltzer M, Ghitani N, Chesler AT (2018) A brainstem-spinal circuit controlling nocifensive behavior. Neuron 100:14911503.e3.

Bennett GJ, Xie YK (1988) A peripheral mononeuropathy in rat that produces disorders of pain sensation like those seen in man. Pain 33:87-107.

Benoist JM, Gautron M, Guilbaud G (1999) Experimental model of trigeminal pain in the rat by constriction of one infraorbital nerve: changes in neuronal activities in the somatosensory cortices corresponding to the infraorbital nerve. Exp Brain Res 126:383-398. 
Bernard JF, Alden M, Besson JM (1993) The organization of the efferent projections from the pontine parabrachial area to the amygdaloid complex: a Phaseolus vulgaris leucoagglutinin (PHA-L) study in the rat. J Comp Neurol 329:201-229.

Bianchi R, Corsetti G, Rodella L, Tredici G, Gioia M (1998) Supraspinal connections and termination patterns of the parabrachial complex determined by the biocytin anterograde tract-tracing technique in the rat. J Anat 193:417-430.

Bie B, Pan ZZ (2003) Presynaptic mechanism for anti-analgesic and antihyperalgesic actions of kappa-opioid receptors. J Neurosci 23:7262-7268.

Bushnell MC, Ceko M, Low LA (2013) Cognitive and emotional control of pain and its disruption in chronic pain. Nat Rev Neurosci 14:502-511.

Castro A, Li Y, Raver C, Chandra R, Masri R, Lobo MK, Keller A (2017a) Neuropathic pain after chronic nerve constriction may not correlate with chloride dysregulation in mouse trigeminal nucleus caudalis neurons. Pain 158:1366-1372.

Castro A, Raver C, Li Y, Uddin O, Rubin D, Ji Y, Masri R, Keller A (2017b) Cortical regulation of nociception of the trigeminal nucleus caudalis. J Neurosci 37:11431-11440.

Cechetto DF, Standaert DG, Saper CB (1985) Spinal and trigeminal dorsal horn projections to the parabrachial nucleus in the rat. J Comp Neurol 240:153-160.

Chaplan SR, Bach FW, Pogrel JW, Chung JM, Yaksh TL (1994) Quantitative assessment of tactile allodynia in the rat paw. J Neurosci Methods 53:5563.

Chen Q, Roeder Z, Li MH, Zhang Y, Ingram SL, Heinricher MM (2017) Optogenetic evidence for a direct circuit linking nociceptive transmission through the parabrachial complex with pain-modulating neurons of the rostral ventromedial medulla (RVM). eNeuro 4:ENEURO.0202-17.2017.

Cheng L, Duan B, Huang T, Zhang Y, Chen Y, Britz O, Garcia-Campmany L, Ren X, Vong L, Lowell BB, Goulding M, Wang Y, Ma Q (2017) Identification of spinal circuits involved in touch-evoked dynamic mechanical pain. Nat Neurosci 20:804-814.

Chiang M, Ross S (2017) (168) Optogenetic dissection of lateral parabrachial pathways in pain perception. J Pain 18:S18.

Chiang MC, Bowen A, Schier LA, Tupone D, Uddin O, Heinricher MM (2019) Parabrachial complex: a Hub for pain and aversion. J Neurosci 39:8225-8230

Chieng BC, Christie MJ, Osborne PB (2006) Characterization of neurons in the rat central nucleus of the amygdala: cellular physiology, morphology, and opioid sensitivity. J Comp Neurol 497:910-927.

Corder G, Ahanonu B, Grewe BF, Wang D, Schnitzer MJ, Scherrer G (2019) An amygdalar neural ensemble that encodes the unpleasantness of pain. Science 363:276-281.

Davern PJ (2014) A role for the lateral parabrachial nucleus in cardiovascular function and fluid homeostasis. Front Physiol 5:436.

Davis M (1994) The role of the amygdala in emotional learning. Int Rev Neurobiol 36:225-266.

deCharms RC, Maeda F, Glover GH, Ludlow D, Pauly JM, Soneji D, Gabrieli JD, Mackey SC (2005) Control over brain activation and pain learned by using real-time functional MRI. Proc Natl Acad Sci USA 102:1862618631.

Dixon WJ (1965) The up-and-down method for small samples. J Am Statist Assoc 60:967-978.

Drew GM, Siddall PJ, Duggan AW (2004) Mechanical allodynia following contusion injury of the rat spinal cord is associated with loss of GABAergic inhibition in the dorsal horn. Pain 109:379-388.

Duvarci S, Pare D (2014) Amygdala microcircuits controlling learned fear. Neuron 82:966-980.

Dworkin RH, White R, O'Connor AB, Baser O, Hawkins K (2007) Healthcare costs of acute and chronic pain associated with a diagnosis of herpes zoster. J Am Geriatr Soc 55:1168-1175.

Dworkin RH, O'Connor AB, Kent J, Mackey SC, Raja SN, Stacey BR, Levy RM, Backonja M, Baron R, Harke H, Loeser JD, Treede RD, Turk DC, Wells CD (2013) Interventional management of neuropathic pain: neuPSIG recommendations. Pain 154:2249-2261.

Ehrlich I, Humeau Y, Grenier F, Ciocchi S, Herry C, Lüthi A (2009) Amygdala inhibitory circuits and the control of fear memory. Neuron 62:757-771.

Fadok JP, Krabbe S, Markovic M, Courtin J, Xu C, Massi L, Botta P, Bylund K, Müller C, Kovacevic A, Tovote P, Lüthi A (2017) A competitive inhibitory circuit for selection of active and passive fear responses. Nature 542:96-100.

Finnerup NB, Attal N, Haroutounian S, McNicol E, Baron R, Dworkin RH, Gilron I, Haanpää M, Hansson P, Jensen TS, Kamerman PR, Lund K, Moore A, Raja SN, Rice AS, Rowbotham M, Sena E, Siddall P, Smith BH, Wallace M (2015) Pharmacotherapy for neuropathic pain in adults: a systematic review and meta-analysis. Lancet Neurol 14:162-173.

Friedberg MH, Lee SM, Ebner FF (1999) Modulation of receptive field properties of thalamic somatosensory neurons by the depth of anesthesia. J Neurophysiol 81:2243-2252.

Gauriau C, Bernard JF (2002) Pain pathways and parabrachial circuits in the rat. Exp Physiol 87:251-258.

Geerling JC, Yokota S, Rukhadze I, Roe D, Chamberlin NL (2017) KöllikerFuse GABAergic and glutamatergic neurons project to distinct targets. J Comp Neurol 525:1844-1860.

Gustin SM, Wilcox SL, Peck CC, Murray GM, Henderson LA (2011) Similarity of suffering: equivalence of psychological and psychosocial factors in neuropathic and non-neuropathic orofacial pain patients. Pain 152:825-832.

Guthmann A, Fritschy JM, Ottersen OP, Torp R, Herbert H (1998) GABA, GABA transporters, GABA(A) receptor subunits, and GAD mRNAs in the rat parabrachial and Kölliker-Fuse nuclei. J Comp Neurol 400:229243.

Hajnal A, Norgren R, Kovacs P (2009) Parabrachial coding of sapid sucrose: relevance to reward and obesity. Ann NY Acad Sci 1170:347-364.

Han S, Soleiman MT, Soden ME, Zweifel LS, Palmiter RD (2015) Elucidating an affective pain circuit that creates a threat memory. Cell 162:363-374.

Herrero JF, Laird JM, López-García JA (2000) Wind-up of spinal cord neurones and pain sensation: much ado about something. Prog Neurobiol 61:169-203.

Institute of Medicine Committee on Advancing Pain Research, Care, and Education (2011) Relieving pain in America: a blueprint for transforming prevention, care, education, and research. Washington, DC: National Academies.

Janak PH, Tye KM (2015) From circuits to behaviour in the amygdala. Nature 517:284-292.

Jergova S, Kolesar D, Cizkova D (2008) Expression of c-Fos in the parabrachial nucleus following peripheral nerve injury in rats. Eur J Pain 12:172179.

Jia HG, Rao ZR, Shi JW (1994) An indirect projection from the nucleus of the solitary tract to the central nucleus of the amygdala via the parabrachial nucleus in the rat: a light and electron microscopic study. Brain Res 663:181-190.

Jia HG, Zhang GY, Wan Q (2005) A GABAergic projection from the central nucleus of the amygdala to the parabrachial nucleus: an ultrastructural study of anterograde tracing in combination with post-embedding immunocytochemistry in the rat. Neurosci Lett 382:153-157.

Kim J, Alger BE (2001) Random response fluctuations lead to spurious paired-pulse facilitation. J Neurosci 21:9608-9618.

Krzywinski M, Altman N (2014) Points of significance: nonparametric tests. Nat Methods 11:467-468.

Laird JM, Bennett GJ (1993) An electrophysiological study of dorsal horn neurons in the spinal cord of rats with an experimental peripheral neuropathy. J Neurophysiol 69:2072-2085.

Landis SC, Amara SG, Asadullah K, Austin CP, Blumenstein R, Bradley EW, Crystal RG, Darnell RB, Ferrante RJ, Fillit H, Finkelstein R, Fisher M, Gendelman HE, Golub RM, Goudreau JL, Gross RA, Gubitz AK, Hesterlee SE, Howells DW, Huguenard J, et al. (2012) A call for transparent reporting to optimize the predictive value of preclinical research. Nature 490:187-191.

Langford DJ, Bailey AL, Chanda ML, Clarke SE, Drummond TE, Echols S, Glick S, Ingrao J, Klassen-Ross T, Lacroix-Fralish ML, Matsumiya L, Sorge RE, Sotocinal SG, Tabaka JM, Wong D, van den Maagdenberg AM, Ferrari MD, Craig KD, Mogil JS (2010) Coding of facial expressions of pain in the laboratory mouse. Nat Methods 7:447-449.

Li JN, Sheets PL (2020) Spared nerve injury differentially alters parabrachial monosynaptic excitatory inputs to molecularly specific neurons in distinct subregions of the central amygdala. Pain 161:166-176.

Loggia ML, Mogil JS, Bushnell MC (2008) Experimentally induced mood changes preferentially affect pain unpleasantness. J Pain 9:784-791.

Manion J, Waller MA, Clark T, Massingham JN, Neely GG (2019) Developing modern pain therapies. Front Neurosci 13:1370. 
Mansour A, Fox CA, Meng F, Akil H, Watson SJ (1994) Kappa 1 receptor mRNA distribution in the rat CNS: comparison to kappa receptor binding and prodynorphin mRNA. Mol Cell Neurosci 5:124-144.

Martelli D, Stanić D, Dutschmann M (2013) The emerging role of the parabrachial complex in the generation of wakefulness drive and its implication for respiratory control. Respir Physiol Neurobiol 188:318-323.

Masri R, Keller A (2012) Chronic pain following spinal cord injury. Adv Exp Med Biol 760:74-88.

Matsumoto N, Bester H, Menendez L, Besson JM, Bernard JF (1996) Changes in the responsiveness of parabrachial neurons in the arthritic rat: an electrophysiological study. J Neurophysiol 76:4113-4126.

Meyer-Rosberg K, Kvarnström A, Kinnman E, Gordh T, Nordfors LO, Kristofferson A (2001) Peripheral neuropathic pain: a multidimensional burden for patients. Eur J Pain 5:379-389.

Morisset V, Nagy F (1998) Nociceptive integration in the rat spinal cord: role of non-linear membrane properties of deep dorsal horn neurons. Eur J Neurosci 10:3642-3652.

Morisset V, Nagy F (2000) Plateau potential-dependent windup of the response to primary afferent stimuli in rat dorsal horn neurons. Eur J Neurosci 12:3087-3095

Navratilova E, Ji G, Phelps C, Qu C, Hein M, Yakhnitsa V, Neugebauer V, Porreca F (2019) Kappa opioid signaling in the central nucleus of the amygdala promotes disinhibition and aversiveness of chronic neuropathic pain. Pain 160:824-832.

Neugebauer V (2015) Amygdala pain mechanisms. Handb Exp Pharmacol 227:261-284.

O'Connor AB, Dworkin RH (2009) Treatment of neuropathic pain: an overview of recent guidelines. Am J Med 122:S22-S32.

Okubo M, Castro A, Guo W, Zou S, Ren K, Wei F, Keller A, Dubner R (2013) Transition to persistent orofacial pain after nerve injury involves supraspinal serotonin mechanisms. J Neurosci 33:5152-5161.

Palecek J, Palecková V, Dougherty PM, Carlton SM, Willis WD (1992) Responses of spinothalamic tract cells to mechanical and thermal stimulation of skin in rats with experimental peripheral neuropathy. J Neurophysiol 67:1562-1573.

Paxinos G, Watson C (2013) The rat brain in stereotaxic coordinates. San Diego: Academic

Polgár E, Wright LL, Todd AJ (2010) A quantitative study of brainstem projections from lamina I neurons in the cervical and lumbar enlargement of the rat. Brain Res 1308:58-67.

Pomrenze MB, Millan EZ, Hopf FW, Keiflin R, Maiya R, Blasio A, Dadgar J, Kharazia V, De Guglielmo G, Crawford E, Janak PH, George O, Rice KC, Messing RO (2015) A transgenic rat for investigating the anatomy and function of corticotrophin releasing factor circuits. Front Neurosci 9:487.

Prescott SA (2015) Synaptic inhibition and disinhibition in the spinal dorsal horn. Prog Mol Biol Transl Sci 131:359-383.

Price TJ, Basbaum AI, Bresnahan J, Chambers JF, De Koninck Y, Edwards RR, Ji RR, Katz J, Kavelaars A, Levine JD, Porter L, Schechter N, Sluka KA, Terman GW, Wager TD, Yaksh TL, Dworkin RH (2018) Transition to chronic pain: opportunities for novel therapeutics. Nat Rev Neurosci 19:383-384.

Roeder Z, Chen Q, Davis S, Carlson JD, Tupone D, Heinricher MM (2016) Parabrachial complex links pain transmission to descending pain modulation. Pain 157:2697-2708.

Roy M, Peretz I, Rainville P (2008) Emotional valence contributes to musicinduced analgesia. Pain 134:140-147.

Roy M, Lebuis A, Peretz I, Rainville P (2011) The modulation of pain by attention and emotion: a dissociation of perceptual and spinal nociceptive processes. Eur J Pain 15:10.

Sanabria ER, Wozniak KM, Slusher BS, Keller A (2004) GCP II (NAALADase) inhibition suppresses mossy fiber-CA3 synaptic neurotransmission by a presynaptic mechanism. J Neurophysiol 91:182-193.

Saper CB, Loewy AD (1980) Efferent connections of the parabrachial nucleus in the rat. Brain Res 197:291-317.

Sarhan M, Freund-Mercier MJ, Veinante P (2005) Branching patterns of parabrachial neurons projecting to the central extended amgydala: single axonal reconstructions. J Comp Neurol 491:418-442.

Sotgiu ML, Biella GE (2002) NMDA and strychnine diversely modulate spinal dorsal horn noxious responsiveness in normal rats: potential significance to sensory disorders in neuropathic pain. Somatosens Mot Res 19:18-23.

Sotocinal SG, Sorge RE, Zaloum A, Tuttle AH, Martin LJ, Wieskopf JS, Mapplebeck JC, Wei P, Zhan S, Zhang S, McDougall JJ, King OD, Mogil JS (2011) The Rat Grimace Scale: a partially automated method for quantifying pain in the laboratory rat via facial expressions. Mol Pain 7:55.

Spike RC, Puskár Z, Andrew D, Todd AJ (2003) A quantitative and morphological study of projection neurons in lamina I of the rat lumbar spinal cord. Eur J Neurosci 18:2433-2448.

Sun N, Yi H, Cassell MD (1994) Evidence for a GABAergic interface between cortical afferents and brainstem projection neurons in the rat central extended amygdala. J Comp Neurol 340:43-64.

Ting JT, Daigle TL, Chen Q, Feng G (2014) Acute brain slice methods for adult and aging animals: application of targeted patch clamp analysis and optogenetics. Methods Mol Biol 1183:221-242.

Todd AJ (2015) Plasticity of inhibition in the spinal cord. Handb Exp Pharmacol 227:171-190.

Torruella-Suárez LM, Vandenberg JR, Cogan ES, Tipton GJ, Teklezghi A, Dange K, Patel GK, McHenry JA, Hardaway JA, Kantak PA, Crowley NA, DiBerto JF, Faccidomo SP, Hodge CW, Stuber GD, McElligott ZA (2019) Manipulations of central amygdala neurotensin neurons alter the consumption of ethanol and sweet fluids in mice. J Neurosci 40:632-647.

Traub RD, Colling SB, Jefferys JG (1995) Cellular mechanisms of 4-aminopyridine-induced synchronized after-discharges in the rat hippocampal slice. J Physiol 489:127-140.

Tyssowski KM, Gray JM (2019) Blue light increases neuronal activity-regulated gene expression in the absence of optogenetic proteins. eNeuro 6: ENEURO.0085-19.2019.

Uddin O, Studlack P, Akintola T, Raver C, Castro A, Masri R, Keller A (2018) Amplified parabrachial nucleus activity in a rat model of trigeminal neuropathic pain. Neurobiol Pain 3:22-30.

Unterwald EM, Knapp C, Zukin RS (1991) Neuroanatomical localization of kappa 1 and kappa 2-opioid receptors in rat and guinea pig brain. Brain Res 562:57-65.

Upshur CC, Luckmann RS, Savageau JA (2006) Primary care provider concerns about management of chronic pain in community clinic populations. J Gen Intern Med 21:652-655.

van Hecke O, Austin SK, Khan RA, Smith BH, Torrance N (2014) Neuropathic pain in the general population: a systematic review of epidemiological studies. Pain 155:654-662.

Veinante P, Yalcin I, Barrot M (2013) The amygdala between sensation and affect: a role in pain. J Mol Psychiatry 1:9.

Vos BP, Strassman AM, Maciewicz RJ (1994) Behavioral evidence of trigeminal neuropathic pain following chronic constriction injury to the rat's infraorbital nerve. J Neurosci 14:2708-2723.

Wang D, Tawfik VL, Corder G, Low SA, François A, Basbaum AI, Scherrer $\mathrm{G}$ (2018) Functional divergence of delta and mu-opioid receptor organization in CNS pain circuits. Neuron 98:90-108.e5.

Wilson TD, Valdivia S, Khan A, Ahn HS, Adke AP, Gonzalez SM, Sugimura YK, Carrasquillo Y (2019) Dual and opposing functions of the central amygdala in the modulation of pain. Cell Rep 29:332-346.e5.

Woodhams SG, Chapman V, Finn DP, Hohmann AG, Neugebauer V (2017) The cannabinoid system and pain. Neuropharmacology 124:105-120.

Woolf CJ, King AE (1987) Physiology and morphology of multireceptive neurons with $\mathrm{C}$-afferent fiber inputs in the deep dorsal horn of the rat lumbar spinal cord. J Neurophysiol 58:460-479.

Yasuda K, Raynor K, Kong H, Breder CD, Takeda J, Reisine T, Bell GI (1993) Cloning and functional comparison of kappa and delta-opioid receptors from mouse brain. Proc Natl Acad Sci USA 90:6736-6740.

Yokota S, Oka T, Tsumori T, Nakamura S, Yasui Y (2007) Glutamatergic neurons in the Kölliker-Fuse nucleus project to the rostral ventral respiratory group and phrenic nucleus: a combined retrograde tracing and in situ hybridization study in the rat. Neurosci Res 59:341-346.

Yu K, Garcia da Silva P, Albeanu DF, Li B (2016) Central amygdala somatostatin neurons gate passive and active defensive behaviors. J Neurosci 36:6488-6496.

Zald DH (2003) The human amygdala and the emotional evaluation of sensory stimuli. Brain Res Brain Res Rev 41:88-123.

Zucker RS (1989) Short-term synaptic plasticity. Annu Rev Neurosci 12:1331. 